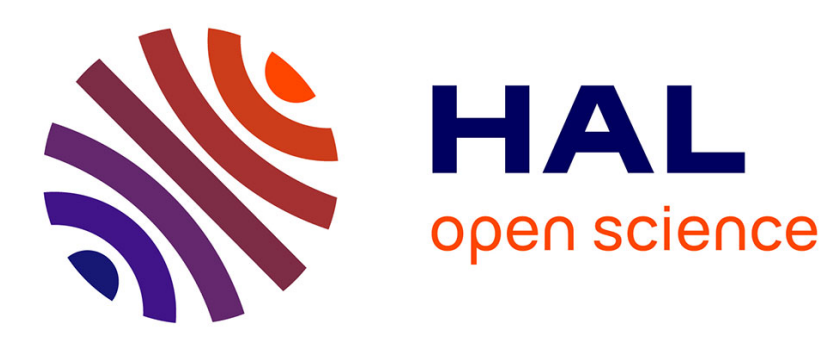

\title{
Optimal selling rules for monetary invariant criteria: tracking the maximum of a portfolio with negative drift,
}

Romuald Elie, Gilles-Edouard Espinosa

\section{To cite this version:}

Romuald Elie, Gilles-Edouard Espinosa. Optimal selling rules for monetary invariant criteria: tracking the maximum of a portfolio with negative drift,. 2012. hal-00573429v2

\section{HAL Id: hal-00573429 \\ https://hal.science/hal-00573429v2}

Preprint submitted on 12 Jul 2012

HAL is a multi-disciplinary open access archive for the deposit and dissemination of scientific research documents, whether they are published or not. The documents may come from teaching and research institutions in France or abroad, or from public or private research centers.
L'archive ouverte pluridisciplinaire HAL, est destinée au dépôt et à la diffusion de documents scientifiques de niveau recherche, publiés ou non, émanant des établissements d'enseignement et de recherche français ou étrangers, des laboratoires publics ou privés. 


\title{
Optimal selling rules for monetary invariant criteria: tracking the maximum of a portfolio with negative drift
}

\author{
Romuald ELIE \\ CEREMADE, CNRS, UMR 7534, \\ Université Paris-Dauphine and CREST \\ elie@ceremade.dauphine.fr
}

\author{
Gilles-Edouard ESPINOSA \\ Department of Mathematics \\ ETH Zurich \\ gilles-edouard.espinosa@math.ethz.ch
}

June 21, 2012

\begin{abstract}
Considering a positive portfolio diffusion $X$ with negative drift, we investigate optimal stopping problems of the form

$$
\inf _{\theta} \mathbb{E}\left[f\left(\frac{X_{\theta}}{\sup _{s \in[0, \tau]} X_{s}}\right)\right],
$$

where $f$ is a non-increasing function, $\tau$ is the next random time where the portfolio $X$ crosses zero and $\theta$ is any stopping time smaller than $\tau$. Hereby, our motivation is the obtention of an optimal selling strategy minimizing the relative distance between the liquidation value of the portfolio and its highest possible value before it reaches zero. This paper unifies optimal selling rules observed by [5] for quadratic absolute distance criteria with bang-bang type ones observed in $[1,4,9]$. More precisely, we provide a verification result for the general stopping problem of interest and derive the exact solution for two classical criteria $f$ of the literature. For the power utility criterion $f: y \mapsto-y^{\lambda}$ with $\lambda>0$, instantaneous selling is always optimal, which is consistent with the observations of [1] or [9] for the Black-Scholes model in finite horizon. On the contrary, for a relative quadratic error criterion, $f: y \mapsto(1-y)^{2}$, selling is optimal as soon as the process $X$ crosses a specified function $\varphi$ of its running maximum $X^{*}$. These results reinforce the idea that optimal stopping problems of similar type lead easily to selling rules of very different nature. Nevertheless, our numerical experiments suggest that the practical optimal selling rule for the relative quadratic error criterion is in fact very close to immediate selling.
\end{abstract}

Key words: optimal stopping, optimal prediction, running maximum, free boundary PDE, verification

MSC Classification (2000): 60G40, 91B28, 35R35 


\section{Introduction}

At a first glance, trying to liquidate a portfolio as close as possible to its ultimate maximum may be viewed as a hopeless ambition for a fund manager. From a mathematical point of view, the random time of interest is not a stopping time. Nevertheless, for a given criterion choice, this kind of optimal prediction problem has remarkably already been addressed in the literature. Graversen, Peskir and Shiryaev were the first authors who tackled successfully this challenging problem. Considering a portfolio $W$ with Brownian motion dynamics on the time interval $[0,1]$, they solve in [6] the optimal stopping problem $\inf _{\theta} \mathbb{E}\left[\left|W_{\theta}-W_{1}^{*}\right|^{2}\right]$, where $W_{1}^{*}$ denotes the maximum of the Brownian motion $W$ at time 1 and $\theta$ is any stopping time smaller than 1. Selling is optimal as soon as the drawdown of the portfolio, i.e. the gap between its current maximum and its value, goes below the function $t \mapsto c^{*} \sqrt{1-t}$, for a specified constant $c^{*}$. Urusov [10] observes that this strategy provides also a good approximation of the last time $\tau^{*}$ where the portfolio reaches its maximum, since it solves the

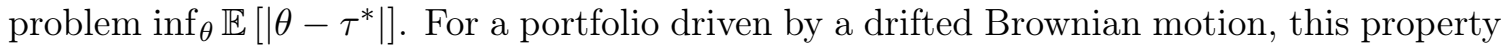
is no longer satisfied, and Du Toit and Peskir [2,3] characterize both solutions of these problems. Once again, stopping is optimal as soon as the drawdown of the portfolio enters a time-to-horizon dependent region.

Considering instead a portfolio composed by one stock $\left(S_{t}\right)_{0 \leq t \leq 1}$ with Black-Scholes dynamics, several authors (Shiryaev, Xu and Zhou [9], Du Toit and Peskir [4] or Dai, Jin, Zhong and Zhou [1]) tried to minimize the relative distance between the stopped stock $S_{\theta}$ and its ultimate maximum $S_{1}^{*}$. In particular they characterize the optimal selling rule associated to the natural stopping problem $\sup _{\theta} \mathbb{E}\left[S_{\theta} / S_{1}^{*}\right]$. As pointed out in [4], the formulation in terms of ratio between the stopped process and its maximum has the effect of stripping away the monetary value of the stock, focusing only on the underlying randomness. Using either probabilistic or deterministic methods, the common interpretation of the solution derived in these papers is that one should "sell bad stocks and keep good ones". Indeed, introducing the "goodness index" $\alpha$ of the stock as the ratio between its excess return rate and its square volatility rate, the optimal strategy appears to be of "bang-bang" type: one should immediately sell the stock if $\alpha \leq 1 / 2$ and keep it until maturity otherwise. Focusing also on the problem $\inf _{\theta} \mathbb{E}\left[S_{1}^{*} / S_{\theta}\right]$, Du Toit and Peskir [4] observe that one should sell immediately if $\alpha<0$, keep until the end if $\alpha>1$ and stop as soon as the ratio $S^{*} / S$

hits a specified deterministic function of time in the intermediate case. It is worth noticing that these two optimal prediction problems of similar type offer therefore different optimal selling strategies for a large range of parameter set.

Of course, the only consideration of stocks with Black-Scholes dynamics is unrealistic and limitative. A recent paper of Espinosa and Touzi [5] allows for the consideration of more general diffusion dynamics and, as a by product, requires to focus on a stationary version of this problem. As pointed out by the authors, considering the first hitting of zero does 
not only have the advantage of reducing the problem to a stationary one, but in fact corresponds to a classical mean-reversion strategy of fund managers. They want to detect the maximum of a signal on each excursion above its mean or symmetrically detect its minimum on each excursion below its mean. Such a signal can be for example generated by the difference between two (normalized) stocks of the same sector. Considering a diffusion portfolio $X$ with general dynamics and negative drift starting at $X_{0}>0$, the authors in [5] study the infinite time horizon problem: $\inf _{\theta} \mathbb{E}\left[\left|X_{\tau}^{*}-X_{\theta}\right|^{2}\right]$ where $\tau$ is the first time where $X$ hits zero and $\theta$ is any stopping time smaller than $\tau$. They solve explicitly this stationary problem as a free boundary one and verify that the fund manager should sell the portfolio whenever its running maximum $X^{*}$ and its drawdown $X^{*}-X$ are both large enough. It is worth noticing that this optimal selling rule differs a lot from the immediate optimal selling strategy obtained by $[1,4,9]$ for the finite time horizon optimal stopping problem $\sup _{\theta} \mathbb{E}\left[X_{\theta} / X_{1}^{*}\right]$, whenever $X$ is a Black-Scholes portfolio with negative drift. Indeed, the nature of optimal selling is very different in the finite horizon case between an absolute and a relative criterion, so it is natural to wonder if this phenomenon also exists in this more realistic stationary framework. The underlying question, which is very important for a fund manager, is whether or not the optimal selling rule is robust with respect to the criterion. Indeed, if two criteria seem very close and are relevant, then they should bring close allocation strategies (here selling/buying rules), because there is no a priori reason to choose one instead of the other.

Therefore the motivation for this article is twofold. First we want to address the same questions as the ones in $[1,4,9]$ but in a context more adapted to fund managers considerations, and then we intend to discuss the robustness of the solution with respect to the criterion. In order to better understand the consequences of the chosen criterion properties on the corresponding optimal selling strategy and hereby unify the corresponding literature, the purpose of this paper is to investigate the optimal selling rules associated to monetary invariant criteria in the stationary framework developed in [5]. The consideration of the ratio between the stopped portfolio and its upcoming maximum allows to capture the scale of the prices themselves and we focus more precisely on the two following problems:

$$
V_{1}=\sup _{\theta} \mathbb{E}\left[\left(\frac{X_{\theta}}{X_{\tau}^{*}}\right)^{\lambda}\right], \quad \text { for } \lambda>0, \quad \text { and } \quad V_{2}=\inf _{\theta} \mathbb{E}\left[\left(\frac{X_{\tau}^{*}-X_{\theta}}{X_{\tau}^{*}}\right)^{2}\right],
$$

where $\tau$ is the first time where $X$ hits 0 and $\theta$ is any stopping time smaller than $\tau$. The first problem consists in maximizing the power utility of the possible relative value of the portfolio. The case $\lambda=1$ corresponds to the criterion considered in $[1,4,9]$ in the finite horizon Black-Scholes framework. Solving the second problem consists simply in minimizing the classical relative quadratic error between the stopped portfolio and its maximum possible value. From the fund manager point of view, choosing between these two criteria seems a priori arbitrary, but we verify hereafter that they lead to optimal selling rules of very different nature. 
For the first problem $V_{1}$, we prove that the optimal stopping strategy consists in liquidating the portfolio immediately. For $\lambda \leq 1$, immediate selling is still optimal even if the portfolio value starts at a point below its current maximum. This is no longer the case when $\lambda>1$, where the fund manager should wait until the value of the portfolio gets close enough to its running maximum. These conclusions extend and are in accordance with the conclusions of $[1,4,9]$ obtained in the finite horizon Black-Scholes framework for $\lambda=1$. Conversely for $V_{2}$, when minimizing the relative quadratic distance between the portfolio and its ultimate maximum, the optimal selling time is the first time where the process $X$ goes below a specified function $\varphi$ of its running maximum $X^{*}$. Similarly to [8] or [5], this function $\varphi$ (or more precisely its inverse) is characterized as the "biggest" solution of an ordinary differential equation and can easily be approximated numerically. Even though for $V_{2}$, the solution seems to be of the same nature as the one in [5], they in fact differ a lot in practice since here $\varphi(0)=0$ whereas $\varphi$ is bounded from below by a positive constant in [5]. For the absolute criterion considered in [5], this implies that one should hold the portfolio no matter how small its value gets, as long as the running maximum of the portfolio has not reached a given minimal threshold. It is worth noticing that this is not the case here.

As already observed by Du Toit and Peskir [4], our results confirm that optimal prediction problems of similar nature can lead to very different types of optimal solution. Predicting the maximum of a portfolio is really intricate and the corresponding optimal selling rule strongly depends on the criterion choice of the fund manager. However, we shall temper a bit this conclusion in our framework, since numerical experiments provided hereafter show that the function $\varphi$ is close to the identity function. Hence, even if immediate stopping is not optimal, a fund manager will not wait long until the drawdown of the portfolio $X^{*}-X$ goes above $X^{*}-\varphi\left(X^{*}\right)$. Thus, immediate selling is for both criteria a reasonable strategy. In addition, the difference between the present result and the one in [5] can also be partly explained. When looking at an absolute criterion, in a certain sense, one neglects the effects related to very small portfolio values in comparison to higher ones. This is why it is not surprising to find a stopping boundary which is bounded from below. On the contrary, the relative criterion penalizes in the same way excursions with a small maximum as excursions with a large maximum. Therefore it is not very surprising to have $\varphi(0)=0$.

The paper is organized as follows. The next section provides the set up of the problem and derives preliminary properties. Section 3 is dedicated to the obtention of a general verification theorem allowing to treat the first and the second optimal stopping problems at once. Sections 4 and 5 tackle successively the power utility type criterion and the quadratic distance one. In both cases, the value function solution is presented and discussed at the beginning of the section, numerical results are provided, and the technical proofs are postponed to the end of it. 


\section{Optimal liquidation of a portfolio}

\subsection{The optimal stopping problem of interest}

Let $W$ be a scalar Brownian motion on the complete probability space $(\Omega, \mathcal{F}, \mathbb{P})$, and denote by $\mathbb{F}=\left\{\mathcal{F}_{t}, t \geq 0\right\}$ the corresponding augmented natural filtration. Let $X$ be a diffusion process given by the following dynamics:

$$
d X_{t}=-\mu\left(X_{t}\right) d t+\sigma\left(X_{t}\right) d W_{t}, \quad t \geq 0
$$

together with an initial data $x:=X_{0}>0$, where $\mu$ and $\sigma$ are Lipschitz continuous functions. We will assume that the portfolio process $X$ is directed towards the origin in the sense that:

$$
\mu(x) \geq 0, \quad \text { for } x \geq 0 .
$$

We denote by $\tau:=\inf \left\{t \geq 0, X_{t}=0\right\}$ the first time where the process $X$ hits the origin, $\mathcal{T}$ the set of $\mathbb{F}$-stopping times $\theta$ such that $\theta \leq \tau$ a.s and $X^{*}:=\sup _{s \leq} X_{s}$ the running maximum of the portfolio $X$. We consider the following optimization problem:

$$
V_{0}:=\inf _{\theta \in \mathcal{T}} \mathbb{E} f\left(\frac{X_{\theta}}{X_{\tau}^{*}}\right)
$$

where $f$ is a non-increasing continuous function on $[0,1], C^{1}$ on $(0,1]$, and such that, there exist two constants $A>0$ and $\eta>0$ satisfying

$$
\left|f^{\prime}(x)\right| \leq A x^{\eta-2}, \quad 0<x \leq 1
$$

Since $f$ is non-increasing, solving this optimal stopping problem consists in minimizing the relative distance, quantified according to the criterion $f$, between the liquidation price of the portfolio and its highest possible value before hitting 0 . We intend to focus in Sections 4 and 5 on two particular classical cases of criteria $f: y \mapsto-y^{\lambda}, \lambda>0$ and $y \mapsto(1-y)^{2}$. Before doing so, we first exhibit preliminary properties and provide a verification argument for the optimal stopping problem (2.3) in its general form.

In order to use dynamic programming techniques, we introduce as usual the process $Z$ defined by $Z_{t}:=z \vee X_{t}^{*}$ for a given $z>0$, and define the corresponding value function associated to the optimization problem (2.3) :

$$
V(x, z):=\inf _{\theta \in \mathcal{T}} \mathbb{E}_{x, z} f\left(\frac{X_{\theta}}{Z_{\tau}}\right), \quad(x, z) \in \Delta
$$

where $\Delta$ is defined by

$$
\boldsymbol{\Delta}:=\{(x, z), 0 \leq x \leq z \text { and } z>0\}
$$

and corresponds to the domain where $(X, Z)$ lies. 
Remark 2.1 Notice that the definition of $\boldsymbol{\Delta}$ differs from the one in [5]. Observe also that contrary to [5], the problem is not invariant by translation. More precisely, if one considers the criterion $\inf _{\theta} f\left(\frac{b+X_{\theta}}{b+Z_{\tau}}\right)$, the problem might not be well-defined for $b<0$ since we can have $b+Z_{\tau}=0$. For $b>0$ (or $z>-b$ if $b<0$ ) however, the problem makes sense and could be studied in a similar fashion, but is not a particular case of what we do here.

Defining the reward function $g$ from immediate stopping

$$
g(x, z):=\mathbb{E}_{x, z} f\left(\frac{x}{Z_{\tau}}\right), \quad(x, z) \in \boldsymbol{\Delta}
$$

observe from the dynamics of $(X, Z)$ that

$$
\mathbb{E}_{x, z} f\left(\frac{X_{\theta}}{Z_{\tau}}\right)=\mathbb{E}_{x, z} \mathbb{E}_{X_{\theta}, Z_{\theta}} f\left(\frac{X_{\theta}}{Z_{\tau}}\right)=\mathbb{E}_{x, z} g\left(X_{\theta}, Z_{\theta}\right), \quad(x, z) \in \boldsymbol{\Delta}, \quad \theta \in \mathcal{T} .
$$

We may thus rewrite this problem in the standard form of an optimal stopping problem

$$
V(x, z)=\inf _{\theta \in \mathcal{T}} \mathbb{E}_{x, z} g\left(X_{\theta}, Z_{\theta}\right), \quad(x, z) \in \boldsymbol{\Delta}
$$

\subsection{Assumptions and first properties}

Let introduce the so-called scale function $S$ defined, for $x \geq 0$, by

$$
S(x):=\int_{0}^{x} e^{\int_{0}^{u} \alpha(r) d r} d u, \quad \text { with } \quad \alpha:=\frac{2 \mu}{\sigma^{2}} .
$$

Remark 2.2 Since the portfolio process $X$ is directed towards 0, the function $\alpha$ is nonnegative. Therefore, the scale function $S$ is increasing, convex and dominates the Identity function.

By construction, $S$ satisfies $S_{x x}=\alpha S_{x}$ and is related to the law of $Z_{\tau}$ via the estimate

$$
\mathbb{P}_{x, z}\left[Z_{\tau} \leq u\right]=\mathbb{P}_{x}\left[X_{\tau}^{*} \leq u\right] \mathbf{1}_{z \leq u}=\left(1-\frac{S(x)}{S(u)}\right) \mathbf{1}_{z \leq u}, \quad(x, z) \in \boldsymbol{\Delta}, \quad u>0 .
$$

Using the scale function $S$, the reward function $g$ rewrites as

$$
g(x, z)=f\left(\frac{x}{z}\right)\left(1-\frac{S(x)}{S(z)}\right)+S(x) \int_{z}^{\infty} f\left(\frac{x}{u}\right) \frac{S^{\prime}(u)}{S(u)^{2}} d u, \quad(x, z) \in \boldsymbol{\Delta},
$$

which is well-defined since $f$ is continuous on $[0,1]$ so that we have

$$
\int_{z}^{\infty}\left|f\left(\frac{x}{u}\right)\right| \frac{S^{\prime}(u)}{S(u)^{2}} d u \leq\|f\|_{\infty} \int_{z}^{\infty} \frac{S^{\prime}(u)}{S(u)^{2}} d u=\frac{\|f\|_{\infty}}{S(z)}, \quad(x, z) \in \boldsymbol{\Delta} .
$$

Via an integration by parts, we deduce

$$
g(x, z)=f\left(\frac{x}{z}\right)-x S(x) \int_{z}^{\infty} f^{\prime}\left(\frac{x}{u}\right) \frac{d u}{u^{2} S(u)}, \quad(x, z) \in \Delta, x>0 .
$$


Observe that the previous integral is well defined since, combining Remark 2.2 with estimate (2.4), we compute

$$
\left|\frac{f^{\prime}\left(\frac{x}{u}\right)}{u^{2} S(u)}\right| \leq A\left(\frac{x}{u}\right)^{\eta-2} \frac{1}{u^{3}}=A \frac{x^{\eta-2}}{u^{1+\eta}}, \quad 0<x \leq u .
$$

If $f$ is $C^{1}$ on $[0,1]$, since $g(0, z)=f(0),(2.11)$ also holds true for $x=0$ and $z>0$.

In this paper, we aim at considering a general framework including the classical types of mean reverting portfolio processes. In particular, we intend to treat the following diffusion dynamics:

- Brownian motion with negative drift: $\alpha$ constant, positive and $S(x)=\frac{e^{\alpha x}-1}{\alpha}$;

- Cox-Ingersol-Ross process: $d X_{t}=-\mu X_{t} d t+\sigma \sqrt{X_{t}+b} d W_{t}$ for some positive constants $\mu, \sigma, b$ so we get $\alpha(x)=\frac{\alpha x}{x+b}$ and $S^{\prime}(x)=e^{\alpha x}\left(\frac{b}{x+b}\right)^{\alpha b}$ with $\alpha>0$ and $b>0$;

- Ornstein-Uhlenbeck process: $d X_{t}=-\mu X_{t} d t+\sigma d W_{t}$ for some positive constants $\mu, \sigma$ so we get $\alpha(x)=\alpha x$ and $S^{\prime}(x)=e^{\alpha x^{2} / 2}$.

For this purpose, we impose on $\alpha$ similar but less restrictive conditions as in [5] and work under the following standing assumption:

$$
\alpha=\frac{2 \mu}{\sigma^{2}}:(0, \infty) \rightarrow \mathbb{R} \text { is a } C^{2} \text { positive non-decreasing concave function. }
$$

Remark 2.3 As in [5], we observe for later use that the restriction (2.12) implies in particular that the function $2 S^{\prime}-\alpha S-2$ is a non-negative increasing function.

Remark 2.4 One can reasonably wonder if the solution of the present problem can be deduced from [5] using the following change of variable: $Y:=\ln (1+X)$, since $Y$ would also be a process directed towards 0 if $X$ is. We claim that this is not the case. Indeed, we can observe that $f\left(\frac{1+X_{\theta}}{1+Z_{\tau}}\right)=f \circ \exp \left(\ln \left(1+X_{\theta}\right)-\ln \left(1+Z_{\tau}\right)\right)$, and define $\ell: x \mapsto f \circ \exp (-x)$. First, as briefly explained in Remark 2.1, the problem considered here where $b=0$ cannot be deduced from the one with $b=1$. Moreover, for the functions $f$ that we intend to study, $f: x \mapsto-x^{\lambda}$ or $f: x \mapsto \frac{1}{2}(1-x)^{2}$, the convexity of $\ell$ required in [5] is not satisfied. Finally, if $X$ is for example an Ornstein-Uhlenbeck process, one can compute that the function $\alpha_{Y}$ associated to $Y$ is of the form $\alpha_{Y}: y \mapsto 2 \alpha\left(e^{2 y}-e^{y}\right)+1$, which is convex on $\mathbb{R}_{+}$, and therefore does not satisfy the assumptions of [5].

\section{A PDE verification argument}

This section is devoted to the obtention of a PDE characterization for the solution of the optimal stopping problem of interest (2.5). We first derive the corresponding HJB equation and then provide a verification theorem. 


\subsection{The corresponding dynamic programming equation}

The linear second order Dynkin operator associated to the diffusion (2.1) is simply given by

$$
\mathcal{L}: v \mapsto v_{x x}-\alpha(x) v_{x}, \quad \text { with } \quad \alpha(x)=\frac{2 \mu(x)}{\sigma^{2}(x)}, \quad \text { for } x \geq 0
$$

By construction, observe that the scale function $S$ satisfies in particular $\mathcal{L} S=0$. Since the value function of interest $V$ rewrites as the solution of a classical optimal stopping problem (2.8), we expect $V$ to be solution of the associated dynamic programming equation. Namely, $V$ should be a solution of the Hamilton-Jacobi-Bellman equation:

$$
\min (\mathcal{L} v ; g-v)=0 ; \quad v(0, z)=f(0), \quad v_{z}(z, z)=0, \quad(x, z) \in \boldsymbol{\Delta}
$$

The first term indicates that $V$ is dominated by the immediate reward function $g$ and that the dynamics of $v$ in the domain are given by the Dynkin operator of the diffusion $X$. The second relation manifests that only immediate stopping is possible whenever the diffusion $X$ has reached 0 . Finally, the last one is the classical Von Neumann condition encountered whenever the diffusion process hits its maximum.

As in any optimal stopping problem, the domain of definition $\boldsymbol{\Delta}$ of the value function subdivides into two subsets: the stopping region $\mathcal{S}$ where immediate liquidation of the portfolio is optimal and the continuation region where the optimal strategy consists in waiting until the portfolio process enters the stopping region. The optimal stopping time is the first time where the process arrives in the stopping region, and, in order to obtain a stopping time in $\mathcal{T}$, we expect the region $\mathcal{S}$ to be a closed subset of $\boldsymbol{\Delta}$. Of course, the stopping region is characterized by the relation $v=g$ since $g$ is the reward function from immediate stopping. Depending on the position of $(x, z) \in \boldsymbol{\Delta}$ with respect to the region $\mathcal{S}$, we expect the dynamics of (3.13) to rewrite

On the stopping region: $\quad v(x, z)=g(x, z), \quad \mathcal{L} g(x, z) \geq 0$

On the continuation region: $\quad v(x, z)<g(x, z), \quad \mathcal{L} v(x, z)=0$;

$$
\text { Everywhere: } \quad v_{z}(x, z) \mathbf{1}_{\{x=z\}}=0 .
$$

Observe that the last Neumann condition is expected on all the domain $\Delta$, and thus surprisingly in the stopping region where the portfolio $X$ does not diffuse. This feature is particular to our framework, in which the reward function $g$ given in (2.10) satisfies by construction $g_{z}(z, z)=0$ for $z>0$.

In the next sections of the paper, we exhibit different shapes of stopping and continuation regions depending on the choice of the objective function $f$. We observe that, although the objective functions may appear rather similar, the optimal selling strategies can be very different. 


\subsection{The verification theorem}

As detailed above, we expect the value function $V$ given by (2.5) to be solution of the Hamilton-Jacobi-Bellman equation (3.13). The solution of this problem is intimately related to the form of the associated stopping region $\mathcal{S}$. Afterwards, we shall not prove that $V$ is indeed a (weak) solution of this PDE but instead try to guess a regular solution to the PDE and verify that it satisfies the assumptions of the following verification theorem.

We first introduce the definition of piecewise $\mathcal{C}^{2,1}$ functions.

Definition 3.1 We say that a function $v$ defined on $\boldsymbol{\Delta}$ is piecewise $\mathcal{C}^{2,1}$ on a subset $E \subset \boldsymbol{\Delta}$ if for any subset $K$ which is compact in $\boldsymbol{\Delta}$, there exist $K_{1}, \ldots, K_{n}$ compacts in $\boldsymbol{\Delta}$ such that $K \cap E \subset \cup_{i=1}^{n} K_{i}$ and $v$ can be extended as a $\mathcal{C}^{2,1}$ function on $K_{i}$ for each $i=1, \ldots, n$.

Theorem 3.1 Let $v$ be a bounded from below function $\mathcal{C}^{1,0}$ on $\boldsymbol{\Delta}$ and piecewise $\mathcal{C}^{2,1}$ on $\boldsymbol{\Delta} \backslash\{(0, z), z>0\}$ in the sense of Definition 3.1.

(i) If $v$ satisfies $\mathcal{L} v \geq 0, v \leq g$ as well as $v_{z}(z, z) \geq 0$ for $z>0$, then $v \leq V$.

(ii) More precisely, if $v_{z}(z, z)=0$ for $z>0$ and there exists a closed in $\Delta$ set $\mathcal{S} \subset \boldsymbol{\Delta}$ containing the axis $\{(0, z), z>0\}$ such that

$$
v=g \text { on } \mathcal{S}, \quad \mathcal{L} v \geq 0 \text { on } \mathcal{S} \backslash\{(0, z), z>0\}, \quad v \leq g \text { and } \mathcal{L} v=0 \text { on } \boldsymbol{\Delta} \backslash \mathcal{S},
$$

then $v=V$ and $\theta^{*}:=\inf \left\{t \geq 0,\left(X_{t}, Z_{t}\right) \in \mathcal{S}\right\}$ is an optimal stopping time.

(iii) If in addition $v<g$ on $\boldsymbol{\Delta} \backslash \mathcal{S}$, then $\theta^{*}$ is the "smallest" optimal stopping time, in the sense that $\theta^{*} \leq \nu$ a.s. for any optimal stopping time $\nu$.

Proof. We prove each assertion separately.

(i) Fix $\left(X_{0}, Z_{0}\right):=(x, z) \in \boldsymbol{\Delta}$. Let $\theta \in \mathcal{T}$ and define $\theta_{n}=n \wedge \theta \wedge \inf \left\{t \geq 0 ; Z_{t} \geq n\right.$ or $\left.Z_{t} \leq \frac{1}{n}\right\}$ for $n \in \mathbb{N}$. Since $(X, Z)$ takes value in a compact subset of $\boldsymbol{\Delta}$, a direct application of Itô's formula gives

$v(x, z)=v\left(X_{\theta_{n}}, Z_{\theta_{n}}\right)-\int_{0}^{\theta_{n}} \mathcal{L} v\left(X_{t}, Z_{t}\right) \frac{\sigma\left(X_{t}\right)^{2}}{2} d t-\int_{0}^{\theta_{n}} v_{x}\left(X_{t}, Z_{t}\right) \sigma\left(X_{t}\right) d W_{t}-\int_{0}^{\theta_{n}} v_{z}\left(X_{t}, Z_{t}\right) d Z_{t}$.

Combining estimates $\mathcal{L} v \geq 0$ and $v_{z}\left(X_{t}, Z_{t}\right) d Z_{t}=v_{z}\left(Z_{t}, Z_{t}\right) d Z_{t} \geq 0$ with the fact that $(X, Z)$ lies in a compact subset of $\boldsymbol{\Delta}$, we deduce

$$
v(x, z) \leq \mathbb{E}_{x, z} v\left(X_{\theta_{n}}, Z_{\theta_{n}}\right) .
$$

Since $v \leq g$, this leads directly to

$$
v(x, z) \leq \mathbb{E}_{x, z} g\left(X_{\theta_{n}}, Z_{\theta_{n}}\right)=\mathbb{E}_{x, z} \mathbb{E}_{X_{\theta_{n}}, Z_{\theta_{n}}} f\left(\frac{X_{\theta_{n}}}{Z_{\tau}}\right)=\mathbb{E}_{x, z} f\left(\frac{X_{\theta_{n}}}{Z_{\tau}}\right) .
$$

Clearly as $n \rightarrow \infty, \theta_{n} \rightarrow \theta$ almost surely. Since $0 \leq X_{\theta_{n}} / Z_{\tau} \leq 1$ and $f$ is continuous, Lebesgue's dominated convergence theorem gives: $\mathbb{E}_{x, z} f\left(X_{\theta_{n}} / Z_{\tau}\right) \rightarrow_{n \rightarrow \infty} \mathbb{E}_{x, z} f\left(X_{\theta} / Z_{\tau}\right)$, leading to

$$
v(x, z) \leq V(x, z) \quad, \quad(x, z) \in \boldsymbol{\Delta} .
$$


(ii) Observe that this framework is more restrictive than the previous one, so that $v \leq V$ on $\boldsymbol{\Delta}$. For $(x, z) \in \mathcal{S}$, we have $v(x, z)=g(x, z) \geq V(x, z)$ by definition of $g$. We now fix $(x, z) \in \boldsymbol{\Delta} \backslash \mathcal{S}$ and prove that $v(x, z) \geq V(x, z)$.

Let $\theta^{*}:=\inf \left\{t \geq 0 ;\left(X_{t}, Z_{t}\right) \in \mathcal{S}\right\}$. Observe that $\theta^{*} \in \mathcal{T}$ since $\mathcal{S}$ is closed in $\Delta$ and contains the axis $\{(0, z), z \geq 0\}$. The regularity of $v$ implies $\mathcal{L} v\left(X_{t}, Z_{t}\right)=0$ for any $t \in\left[0, \theta^{*}\right)$. As before, we define $\theta_{n}^{*}:=n \wedge \theta^{*} \wedge \inf \left\{t \geq 0 ; Z_{t} \geq n\right.$ or $\left.Z_{t} \leq \frac{1}{n}\right\}$, which is a stopping time. A very similar computation leads directly to

$$
v(x, z)=\mathbb{E}_{x, z} v\left(X_{\theta_{n}^{*}}, Z_{\theta_{n}^{*}}\right) .
$$

Since $v$ is bounded from below and $v \leq g \leq\|f\|_{\infty}, v$ is bounded. Therefore the sequence $\left(v\left(X_{\theta_{n}^{*}}, Z_{\theta_{n}^{*}}\right)\right)_{n}$ is uniformly integrable and we deduce that $v(x, z)=\mathbb{E}_{x, z} v\left(X_{\theta^{*}}, Z_{\theta^{*}}\right)$. Since $\left(X_{\theta^{*}}, Z_{\theta^{*}}\right) \in \mathcal{S}$ and $v=g$ on $\mathcal{S}$, we get

$$
v(x, z)=\mathbb{E}_{x, z} g\left(X_{\theta^{*}}, Z_{\theta^{*}}\right)=\mathbb{E}_{x, z} f\left(\frac{X_{\theta^{*}}}{Z_{\tau}}\right) \geq V(x, z) .
$$

Thus $v=V$ on $\boldsymbol{\Delta}$ and $\theta^{*}$ is an optimal stopping time.

(iii) For a given $(x, z) \in \boldsymbol{\Delta}$, we argue by contradiction and suppose the existence of a stopping time $\nu \in \mathcal{T}$ satisfying $\mathbb{P}\left(\nu<\theta^{*}\right)>0$ and $V(x, z)=\mathbb{E}_{x, z} f\left(X_{\nu} / Z_{\tau}\right)$.

By assumption, we have $V\left(X_{\nu}, Z_{\nu}\right)<g\left(X_{\nu}, Z_{\nu}\right)$ on $\left\{\tau<\theta^{*}\right\}$, which combined with estimate $V \leq g$ implies

$$
V(x, z)=\mathbb{E}_{x, z} f\left(\frac{X_{\nu}}{Z_{\tau}}\right)=\mathbb{E}_{x, z} g\left(X_{\nu}, Z_{\nu}\right)>\mathbb{E}_{x, z} V\left(X_{\tau}, Z_{\tau}\right) \geq V(x, z),
$$

where the last inequality follows from the definition of $V$. This leads to a contradiction, which guarantees the minimality of $\theta^{*}$.

Remark 3.1 From the definition of $g$, one easily checks that $g_{z}(z, z)=0$ for any $z>0$. Therefore, in the PDE dynamics (3.14), the Neumann boundary condition $v_{z}(z, z)=0$ is only necessary for $(z, z) \in \boldsymbol{\Delta} \backslash \mathcal{S}$, since it is automatically satisfied otherwise.

Remark 3.2 Whenever $g$ is a function which is $\mathcal{C}^{1,0}$ w.r.t. $(x, z)$ on $\boldsymbol{\Delta}, \mathcal{C}^{2,1}$ w.r.t. $(x, z)$ on $\boldsymbol{\Delta} \backslash\{(0, z), z>0\}$ and $\mathcal{L} g \geq 0$ on $\boldsymbol{\Delta} \backslash\{(0, z), z>0\}$, then $v=g$ and $\mathcal{S}=\boldsymbol{\Delta}$ satisfy the assumptions of Theorem 3.1 (ii). In that case, immediate stopping is always optimal. We prove in Proposition 3.1 that the reverse is true. Notice also that expression (2.10) implies that an immediate sufficient condition for $g$ to be in $\mathcal{C}^{1,0}(\boldsymbol{\Delta}) \cap \mathcal{C}^{2,1}(\boldsymbol{\Delta} \backslash\{(0, z), z>0\})$ is that $f$ is $\mathcal{C}^{2}$ on $(0,1]$.

Proposition 3.1 Assume that $g$ is $\mathcal{C}^{1,0}$ on $\boldsymbol{\Delta}, \mathcal{C}^{2,1}$ on $\boldsymbol{\Delta} \backslash\{(0, z), z>0\}$, and that there exists $\left(x_{0}, z_{0}\right) \in \boldsymbol{\Delta} \backslash\{(0, z), z>0\}$ such that $\mathcal{L} g\left(x_{0}, z_{0}\right)<0$. Then, immediate stopping at $\left(x_{0}, z_{0}\right)$ is not optimal (or equivalently $V\left(x_{0}, z_{0}\right)<g\left(x_{0}, z_{0}\right)$ ). 
Proof. Since $\mathcal{L} g$ is continuous at $\left(x_{0}, z_{0}\right)$, there exists a neighborhood $U_{0}$ of $\left(x_{0}, z_{0}\right)$ in $\boldsymbol{\Delta}$ such that $\mathcal{L} g(x, z)<0$ for any $(x, z) \in U_{0}$. Without loss of generality, we can assume that $U_{0}$ is compact in $\boldsymbol{\Delta}$. Let $\left(X_{0}, Z_{0}\right)=\left(x_{0}, z_{0}\right)$. Since $x_{0}>0$, there exists $\theta_{0} \in \mathcal{T}$ such that $\mathbb{E}_{x_{0}, z_{0}} \theta_{0}>0$ and let define $\theta_{1}:=1 \wedge \theta_{0} \wedge \inf \left\{t \geq 0 ; \quad\left(X_{t}, Z_{t}\right) \notin U_{0}\right\} \in \mathcal{T}$. Since $\left\{\theta_{0}>0\right\}=\left\{\theta_{1}>0\right\}$, we also have $\mathbb{E}_{x_{0}, z_{0}} \theta_{1}>0$. Using Itô's formula, we compute:

$$
\begin{aligned}
g\left(x_{0}, z_{0}\right)=g( & \left.X_{\theta_{1}}, Z_{\theta_{1}}\right)-\int_{0}^{\theta_{1}} \mathcal{L} g\left(X_{u}, Z_{u}\right) \frac{\sigma\left(X_{u}\right)^{2}}{2} d u \\
& -\int_{0}^{\theta_{1}} g_{x}\left(X_{u}, Z_{u}\right) \sigma\left(X_{u}\right) d W_{u}-\int_{0}^{\theta_{1}} g_{z}\left(X_{u}, Z_{u}\right) d Z_{u} .
\end{aligned}
$$

From Remark 3.1, $g_{z}(z, z)=0$ for $z>0$ so that the last term of the previous expression disappears. Since $U_{0}$ is compact and $\mathbb{E}_{x_{0}, z_{0}} \theta_{1}>0$, taking conditional expectations, we deduce that $g\left(x_{0}, z_{0}\right)>\mathbb{E}_{x_{0}, z_{0}} g\left(X_{\theta_{1}}, Z_{\theta_{1}}\right) \geq V\left(x_{0}, z_{0}\right)$.

In the next sections, we investigate two particular cases of objective functions, for which we exhibit functions $v$ and stopping regions $\mathcal{S}$, which satisfy the assumptions of Theorem 3.1 and are in general non trivial.

\section{The power utility criterion}

Let first examine the case where the function $\mathrm{f}$ is given by $f: x \mapsto-\frac{x^{\lambda}}{\lambda}$, for $\lambda>0$. In other words, we are computing the following value function

$$
V^{\lambda}(x, z):=-\frac{1}{\lambda} \sup _{\theta \in \mathcal{T}} \mathbb{E}_{x, z}\left(\frac{X_{\theta}}{Z_{\tau}}\right)^{\lambda}, \quad(x, z) \in \Delta, \quad \lambda>0 .
$$

Consider an investor, whose relative preferences are given by a power utility function and suppose that he detains at time 0 a given portfolio $X$ directed towards 0 . The optimal stopping time at which he should liquidate his portfolio is the solution of the previous prediction problem. With a given finite time horizon $T$, du Toit and Peskir [4] as well as Shiryaev, Xu and Zhou [9] investigate the case where $X$ is a Geometric Brownian motion. They conclude that the optimal strategy consists in waiting until time $T$ if the portfolio has promising returns (i.e. $1<2 \mu / \sigma^{2}=x \alpha(x), x>0$, with our notations), and sell immediately otherwise. In our stationary framework, waiting until the wealth reaches 0 is obviously a non optimal strategy. For a linear utility function $(\lambda=1)$, we prove in Theorem 4.1 below that immediate stopping is also optimal. Depending on the value of $\lambda$, the latter may no longer be the case for the non linear problem (4.16). Nevertheless, we observe that immediate selling is still optimal for the practical value function of interest $V^{\lambda}(x, x)$, for $x>0$.

\subsection{The particular case where $\lambda \leq 1$}

For $\lambda \leq 1$, we prove hereafter that immediate stopping is always optimal. For $\lambda=1$, these conclusions are therefore in accordance with those of $[4,9]$ obtained for the case of an ex- 
ponential Brownian motion on a fixed time horizon.

A direct application of estimate (2.11) proves that the reward function $g^{\lambda}$ associated to problem (4.16) is given by

$$
g^{\lambda}(x, z)=-\frac{x^{\lambda}}{\lambda z^{\lambda}}+x^{\lambda} S(x) \int_{z}^{\infty} \frac{d u}{S(u) u^{1+\lambda}}, \quad(x, z) \in \boldsymbol{\Delta}, \quad \lambda>0 .
$$

The next theorem indicates that the framework of Remark 3.2 holds for $\lambda \leq 1$, so that $g^{\lambda}$ coincides with the value function on $\boldsymbol{\Delta}$.

Theorem 4.1 For $\lambda \leq 1$, immediate stopping is optimal for problem (4.16), so that

$$
V^{\lambda}(x, z)=g^{\lambda}(x, z), \quad(x, z) \in \Delta, \quad 0<\lambda \leq 1 .
$$

Proof. For any $\lambda>0$ and $(x, z) \in \boldsymbol{\Delta}$ with $x>0$, we compute

$$
g_{x}^{\lambda}(x, z)=-\frac{x^{\lambda-1}}{z^{\lambda}}+\left\{\lambda x^{\lambda-1} S(x)+x^{\lambda} S^{\prime}(x)\right\} \int_{z}^{\infty} \frac{d u}{S(u) u^{1+\lambda}}
$$

Differentiating one more time and using the relation $\mathcal{L} S=0$, we get,

$g_{x x}^{\lambda}(x, z)=(1-\lambda) \frac{x^{\lambda-2}}{z^{\lambda}}+\left\{\lambda(\lambda-1) x^{\lambda-2} S(x)+\left(2 \lambda x^{\lambda-1}+x^{\lambda} \alpha(x)\right) S^{\prime}(x)\right\} \int_{z}^{\infty} \frac{d u}{S(u) u^{1+\lambda}}$,

for any $\lambda \neq 1$ and $0<x \leq z$. Combining the previous estimates, we deduce that

$$
\mathcal{L} g^{\lambda}(x, z)=x^{\lambda-2}[x \alpha(x)+1-\lambda]\left(\frac{1}{z^{\lambda}}-\int_{z}^{\infty} \frac{S(x)}{S(u)} \frac{\lambda d u}{u^{1+\lambda}}\right)+2 x^{\lambda-1} S^{\prime}(x) \int_{z}^{\infty} \frac{\lambda d u}{S(u) u^{1+\lambda}},
$$

for any $\lambda \neq 1$ and $0<x \leq z$. In the case, where $\lambda=1$, we get similarly

$$
\mathcal{L} g^{1}(x, z)=\alpha(x)\left(\frac{1}{z}-\int_{z}^{\infty} \frac{S(x) d u}{u^{2} S(u)}\right)+2 S^{\prime}(x) \int_{z}^{\infty} \frac{d u}{u^{2} S(u)}, \quad(x, z) \in \Delta .
$$

Furthermore, since $S$ is increasing, we have

$$
\int_{z}^{\infty} \frac{S(x)}{S(u)} \frac{\lambda d u}{u^{1+\lambda}} \leq \int_{z}^{\infty} \frac{S(z)}{S(u)} \frac{\lambda d u}{u^{1+\lambda}} \leq \int_{z}^{\infty} \frac{\lambda d u}{u^{1+\lambda}}=\frac{1}{z^{\lambda}}, \quad(x, z) \in \boldsymbol{\Delta}, \quad \lambda>0 .
$$

Plugging this estimate in (4.17) and (4.18), we see that $\mathcal{L} g^{\lambda} \geq 0$ on $\boldsymbol{\Delta} \backslash\{(0, z), z>0\}$ for any $\lambda \leq 1$. As detailed in Remark 3.2, since $g$ is $C^{0}$ on $\boldsymbol{\Delta}$ and $C^{2,1}$ on $\boldsymbol{\Delta} \backslash\{(0, z), z>0\}$, we deduce that $V^{\lambda}=g^{\lambda}$ on $\boldsymbol{\Delta}$ and consequently immediate stopping is optimal for any $\lambda \leq 1$. 


\subsection{Construction of the solution when $\lambda>1$}

We now turn to the more interesting and intricate case where $\lambda>1$. Then, the function $\mathcal{L} g^{\lambda}$ is still given by expression (4.17) and we observe that:

$$
\mathcal{L} g^{\lambda}(x, z) \sim_{x \sim 0}(1-\lambda) \frac{x^{\lambda-2}}{z^{\lambda}}<0,
$$

for any $z>0$ and $\lambda>1$. Therefore, $\mathcal{L} g^{\lambda}$ is not non-negative on $\boldsymbol{\Delta}$ and Proposition 3.1 ensures that the associated continuation region is non empty. Since immediate stopping shall not be optimal close to the axis $\{(0, z) ; z>0\}$, we expect to have a stopping region of the form $\mathcal{S}^{\lambda}:=\left\{(x, z) \in \boldsymbol{\Delta} ; x \geq \varphi^{\lambda}(z)\right\} \cup\{(0, z) ; z>0\}$. Hence, our objective is to find functions $v^{\lambda}$ and $\varphi^{\lambda}$ satisfying

$$
\begin{array}{rll}
\mathcal{L} v^{\lambda}(x, z) & =0 \quad \text { for } 0<x<\varphi^{\lambda}(z) \quad \text { and }(x, z) \in \boldsymbol{\Delta}, \\
v^{\lambda}(x, z) & =g^{\lambda}(x, z) \text { and } \mathcal{L} g^{\lambda}(x, z) \geq 0 \quad \text { for } x \geq \varphi^{\lambda}(z) \quad \text { and }(x, z) \in \boldsymbol{\Delta}, \\
v^{\lambda}(0, z) & =0 \quad \text { for } z>0, \\
v_{z}^{\lambda}(z, z) & =0 \quad \text { for } z>0 .
\end{array}
$$

Since we look for regular solutions, we complement the above system by the continuity and the smoothfit conditions

$$
v^{\lambda}\left(\varphi^{\lambda}(z), z\right)=g^{\lambda}\left(\varphi^{\lambda}(z), z\right) \quad \text { and } \quad v_{x}^{\lambda}\left(\varphi^{\lambda}(z), z\right)=g_{x}^{\lambda}\left(\varphi^{\lambda}(z), z\right), \quad \text { for } z>0 .
$$

The stopping region $\mathcal{S}^{\lambda}$ will then be defined as

$$
\mathcal{S}^{\lambda}:=\left\{(x, z) \in \Delta ; x \geq \varphi^{\lambda}(z)\right\} \cup\{(0, z) ; z>0\} .
$$

Since the optimization problem of practical interest corresponds to the value of $V^{\lambda}$ on the diagonal $\{(x, x) ; x>0\}$, our main concern here is to find out if $\varphi^{\lambda}(z) \leq z$ for any $z \geq 0$, hence indicating if immediate stopping is always optimal on the diagonal. Surprisingly, we verify hereafter that $\varphi^{\lambda}(0)=0$ and $\varphi^{\lambda}(z)<z$, for $z>0$, so that immediate stopping is the optimal strategy for the practical problem of interest.

Due to the dynamics of (4.19) and since $\mathcal{L} S=0$, the function $v^{\lambda}$ must be of the form

$$
v^{\lambda}(x, z)=A(z)+B(z) S(x), \quad(x, z) \in \boldsymbol{\Delta} \backslash \mathcal{S} .
$$

Combined with the continuity and smooth fit conditions (4.23), this leads to

$$
v(x, z)=g^{\lambda}\left(\varphi^{\lambda}(z), z\right)+\frac{g_{x}^{\lambda}\left(\varphi^{\lambda}(z), z\right)}{S^{\prime} \circ \varphi^{\lambda}(z)}\left[S(x)-S \circ \varphi^{\lambda}(z)\right], \quad(x, z) \in \boldsymbol{\Delta} \backslash \mathcal{S} .
$$

The free boundary $\varphi^{\lambda}$ is then determined by the Dirichlet condition (4.21) and must satisfy:

$$
g^{\lambda}\left(\varphi^{\lambda}(z), z\right) S^{\prime} \circ \varphi^{\lambda}(z)=g_{x}^{\lambda}\left(\varphi^{\lambda}(z), z\right) S \circ \varphi^{\lambda}(z), \quad(x, z) \in \boldsymbol{\Delta} \backslash \mathcal{S} .
$$

The next lemma introduces a free boundary function $\varphi^{\lambda}$ satisfying this required condition. It also provides useful properties of this free boundary function and its technical proof is postponed to Section 4.4 . 
Lemma 4.1 For any $\lambda>1$, the function $\varphi^{\lambda}$ given by

$$
\varphi^{\lambda} \quad: \quad z \in(0, \infty) \mapsto \arg \min _{x \in[0, z]} \frac{g(x, z)}{S(x)}
$$

is a well defined increasing $C^{1}$ function, satisfying:

(i) $0 \leq \varphi^{\lambda}(z)<z$, for any $z>0$;

(ii) $\varphi^{\lambda}$ maps $(0, \infty)$ onto $\left(0, y^{\lambda}\right)$, where $y^{\lambda}$ is the unique non null zero of $y \mapsto y S^{\prime}(y)-\lambda S(y)$.

Remark 4.1 As pointed out by a referee, the definition of the optimal frontier $\varphi^{\lambda}$ interprets as the one associated to a one-dimensional classical stopping problem with fixed running maximum $z>0$. Indeed, we observe ex-post that the exhibited optimal policy does not allow the running maximum of the portfolio to increase. Hence solving this stopping problem with a fixed $z>0$ is essentially the same.

Remark 4.2 Observe that, for any fixed $z>0$ and $\lambda>1, g^{\lambda}(x, z) / S(x)$ converges to 0 as $x$ goes to 0 , since $S$ dominates the Identity function as pointed out in Remark 2.2. Therefore, the function $g^{\lambda}(., z) / S($.$) is well defined on [0, z]$ for any $z>0$.

Before providing the value function solution and verifying that it satisfies the requirements of Theorem 3.1, we still need to check that the stopping region $\mathcal{S}^{\lambda}$ associated to $\varphi^{\lambda}$ is indeed a good candidate, i.e. that the second part of (4.20) holds. This is the purpose of the next lemma, which proof is also postponed to Section 4.4 .

Lemma 4.2 For any $\lambda>1$, the function $\mathcal{L} g^{\lambda}$ is non-negative on $\left\{(x, z) \in \boldsymbol{\Delta}, x \geq \varphi^{\lambda}(z)\right\}$.

Given the free boundary $\varphi^{\lambda}$ defined above and the corresponding stopping region $\mathcal{S}^{\lambda}$, we are now in position to provide the optimal strategy and value function solutions of the problem (4.16).

Theorem 4.2 For any $\lambda>1$, the value function $V^{\lambda}$ solution of problem (4.16) is given by

$$
V^{\lambda}(x, z)=g^{\lambda}\left(\varphi^{\lambda}(z), z\right) \frac{S(x)}{S \circ \varphi^{\lambda}(z)} \mathbf{1}_{\left\{x<\varphi^{\lambda}(z)\right\}}+g^{\lambda}(x, z) \mathbf{1}_{\left\{x \geq \varphi^{\lambda}(z)\right\}}, \quad(x, z) \in \boldsymbol{\Delta} .
$$

The smallest optimal stopping time associated to (4.16) is given by

$$
\theta^{\lambda}:=\inf \left\{t \geq 0, \quad X_{t} \geq \varphi^{\lambda}\left(Z_{t}\right)\right\} \wedge \tau, \quad \lambda>1
$$

Proof. Let denote by $v^{\lambda}$ the candidate value function defined by the right-hand side of (4.25). We shall prove that $v^{\lambda}$ coincides with the value function (4.16) by checking that it satisfies all the requirements of Theorem 3.1.

It is immediate that $v^{\lambda}$ is bounded from below by 0 because $g^{\lambda} \geq 0$. Since $g^{\lambda}$ is $C^{1}$ on $\boldsymbol{\Delta}$ and $C^{2,1}$ w.r.t. $(x, z)$ on $\boldsymbol{\Delta} \backslash\{(0, z), z>0\}$, and $\varphi^{\lambda}$ is $C^{1}$ by Lemma $4.1, v^{\lambda}$ is $C^{2,1}$ w.r.t. 
$(x, z)$ on both $\boldsymbol{\Delta} \backslash \mathcal{S}$ and $\mathcal{S} \backslash\{(0, z), z>0\}$, so that it is piecewise $C^{2,1}$ on $\boldsymbol{\Delta} \backslash\{(0, z), z>0\}$. By construction, $v^{\lambda}$ is continuous on $\boldsymbol{\Delta}$ and we recall from the definition of $\varphi^{\lambda}$ that

$$
g^{\lambda}\left(\varphi^{\lambda}(z), z\right) \frac{S^{\prime} \circ \varphi^{\lambda}(z)}{S \circ \varphi^{\lambda}(z)}=g_{x}^{\lambda}\left(\varphi^{\lambda}(z), z\right), \quad z>0 .
$$

Therefore, $v^{\lambda}$ is $C^{1}$ on $\boldsymbol{\Delta}$.

The closed in $\Delta$ stopping region associated to the value function $v^{\lambda}$ is naturally given by (4.24). By definition, $v^{\lambda}=g^{\lambda}$ on $\mathcal{S}^{\lambda}$ and we deduce from Lemma 4.2 that $\mathcal{L} g^{\lambda} \geq 0$ on the set $\mathcal{S}^{\lambda} \backslash\{(0, z), z>0\}$. By construction, we have $\mathcal{L} v^{\lambda}=0$ on $\boldsymbol{\Delta} \backslash \mathcal{S}^{\lambda}$. For any $z>0$, since $g^{\lambda}(., z) / S$ achieves its minimum at a unique point $\varphi^{\lambda}(z)$, we get

$$
g^{\lambda}\left(\varphi^{\lambda}(z), z\right) \frac{S(x)}{S \circ \varphi^{\lambda}(z)}<g^{\lambda}(x, z), \quad 0 \leq x<\varphi^{\lambda}(z)
$$

and we deduce that $v^{\lambda}<g^{\lambda}$ on $\boldsymbol{\Delta} \backslash \mathcal{S}^{\lambda}$. Finally, since $v_{z}^{\lambda}(z, z)=g_{z}^{\lambda}(z, z)=0$ for any $z>0$, all the requirements of (ii)-(iii) in Theorem 3.1 are in force, and the proof is complete.

\subsection{Properties of the optimal liquidation strategy}

We first observe that the two previous cases where $\lambda$ is above or below 1 seem to be of different natures. However, we prove hereafter that this is not the case and provide via simple arguments the continuity of $V^{\lambda}$ with respect to the parameter $\lambda$.

Proposition 4.1 The mapping $\lambda \mapsto V^{\lambda}$ is continuous on $(0, \infty)$.

Proof. We fix $\lambda_{1}$ and $\lambda_{2}$ in $(0, \infty)$ such that $\lambda_{1} \leq \lambda_{2}$. First notice that since $X . \leq Z_{\tau}$ on $[0, \tau]$, we necessarily have

$$
-\lambda_{2} V^{\lambda_{2}}(x, z)=\sup _{\theta \in \mathcal{T}} \mathbb{E}_{x, z}\left(\frac{X_{\theta}}{Z_{\tau}}\right)^{\lambda_{2}} \leq \sup _{\theta \in \mathcal{T}} \mathbb{E}_{x, z}\left(\frac{X_{\theta}}{Z_{\tau}}\right)^{\lambda_{1}}=-\lambda_{1} V^{\lambda_{1}}(x, z), \quad(x, z) \in \boldsymbol{\Delta} .
$$

Now, using Jensen's inequality, we observe that

$$
\left[\mathbb{E}_{x, z}\left(\frac{X_{\theta}}{Z_{\tau}}\right)^{\lambda_{1}}\right]^{\frac{\lambda_{2}}{\lambda_{1}}} \leq \mathbb{E}_{x, z}\left(\frac{X_{\theta}}{Z_{\tau}}\right)^{\lambda_{2}} \leq-\lambda_{2} V^{\lambda_{2}}(x, z), \quad \theta \in \mathcal{T}, \quad(x, z) \in \boldsymbol{\Delta} .
$$

Bringing this expression to the power $\lambda_{1} / \lambda_{2}$ and taking the supremum over $\theta$, we deduce from (4.26) that

$$
\left[-\lambda_{1} V^{\lambda_{1}}(x, z)\right]^{\frac{\lambda_{2}}{\lambda_{1}}} \leq-\lambda_{2} V^{\lambda_{2}}(x, z) \leq-\lambda_{1} V^{\lambda_{1}}(x, z), \quad(x, z) \in \boldsymbol{\Delta} .
$$

Therefore $\lambda_{2} V^{\lambda_{2}} \rightarrow \lambda_{1} V^{\lambda_{1}}$ whenever $\lambda_{2} \rightarrow \lambda_{1}$ and we deduce the continuity of $V^{\lambda}$ with respect to $\lambda$. 
For $\lambda>1$, Theorem 4.2 indicates that the stopping region $\mathcal{S}^{\lambda}$ associated to problem (4.16) is given by (4.24). Since $\varphi^{\lambda}(z)<z$, we see that the stopping region $\mathcal{S}^{\lambda}$ includes in particular the axis $\{(x, x), x>0\}$. Therefore, if an investor detains a portfolio directed towards zero and hopes to get close to its upcoming maximum before it reaches zero according to the criterion (4.16), he should liquidate the portfolio immediately. If ever the running maximum $z$ of the portfolio exceeds its current value, he should wait until the value of the portfolio hits zero or increases enough and reaches $\varphi^{\lambda}(z)$. In any case, the optimal strategy does not allow the running maximum of the portfolio to increase. Theorem 4.1 indicates that immediate selling is optimal for $\lambda \in(0,1]$ and these results are in accordance with those of [9] for an exponential Brownian motion on a finite fixed horizon, since waiting until maturity is irrelevant in our framework. Nevertheless, changing the criterion of interest may lead to value functions where immediate stopping is not optimal on the axis $\{(x, x), x>0\}$. This is exactly the purpose of Section 5 .

Free Boundary of OU for different lambda

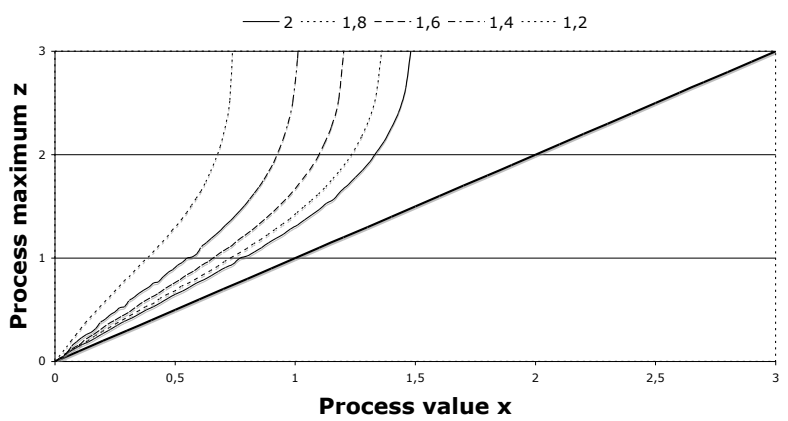

Free Boundary of CIR for different lambda

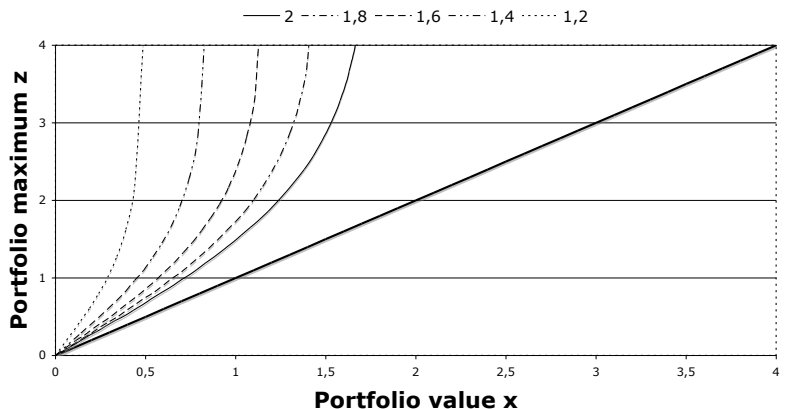

Figure 1: Optimal frontier for an OU $(\alpha=1)$ and a CIR $(\alpha=1, b=1)$ with different parameter $\lambda$

Figure 1 represents the frontier between the stopping and the continuation regions for different values of $\lambda$ larger than 1 and associated to an Orstein-Uhlenbeck with parameter $\alpha=1$ and a CIR-Feller process with parameters $\alpha=1$ and $b=1$. We first observe that the shape of the free boundary $\varphi^{\lambda}$ is rather similar in both cases, and we observe indeed this feature for a large range of parameter set. Furthermore, the mapping $\lambda \mapsto \varphi^{\lambda}$ seems to be continuous, property which is easily verified from the definition of $\varphi^{\lambda}$. Second, we notice that the free boundary $\varphi^{\lambda}$ is decreasing with respect to $\lambda$. Indeed, arguing as in Part 2. of the proof of Lemma 4.1, one can easily check that the function $x \in \mathbb{R}^{+} \mapsto x S^{\prime}(x) / S(x)$ is decreasing starting from 1 . Hence, by definition of $y^{\lambda}$, the valuation domain $\left[0, y^{\lambda}\right]$ of $\varphi^{\lambda}$ shrinks monotonically to $\{0\}$ as $\lambda$ decreases to 1 , hence leading to the absence of continuation region for the problem $V^{1}$. 
Remark 4.3 Considering for example an Ornstein-Uhlenbeck portfolio $X$, one verifies easily from their definitions that the free boundary $\varphi^{\lambda}$ and the value function $v^{\lambda}$ are continuous with respect to the parameter $\alpha \in \mathbb{R}$, characterizing the dynamics of the portfolio $X$. Hence, the continuation and stopping regions are not too sensitive to eventual estimation errors of this parameter of interest.

\subsection{Proofs of Lemma 4.1 and Lemma 4.2}

This section provides successively the proofs of Lemma 4.1 and Lemma 4.2.

\section{Proof of Lemma 4.1}

Fix $\lambda>1$. Let introduce the functions

$$
m: x \mapsto \frac{x S^{\prime}(x)}{\lambda S(x)^{2}}-\frac{1}{S(x)} \quad \text { and } \quad \ell: z \mapsto-\int_{z}^{\infty} \frac{\lambda z^{\lambda} d u}{S(u) u^{1+\lambda}},
$$

so that the derivative of the function of interest rewrites

$$
\frac{\partial}{\partial x}\left[\frac{g(x, z)}{S(x)}\right]=\frac{x^{\lambda-1}}{z^{\lambda}}\{m(x)-\ell(z)\}, \quad(x, z) \in \boldsymbol{\Delta} .
$$

0. A useful estimate.

We will use several times the following expansion as $x \rightarrow \infty$ :

$$
\alpha(x) S(x) \sim_{\infty} S^{\prime}(x)
$$

Indeed, recalling that $\mathcal{L} S=0$ and integrating by parts, we compute:

$S(x)=S(1)+\int_{1}^{x} \frac{\alpha(u) S^{\prime}(u)}{\alpha(u)} d u=S(1)+\frac{S^{\prime}(x)}{\alpha(x)}-\frac{S^{\prime}(1)}{\alpha(1)}-\int_{1}^{x}(1 / \alpha)^{\prime}(u) S^{\prime}(u) d u \sim_{\infty} \frac{S^{\prime}(x)}{\alpha(x)}$,

since $S(x) \rightarrow \infty$ as $x \rightarrow \infty$ and (2.12) implies that $(1 / \alpha)^{\prime}(x) \rightarrow 0$ as $x \rightarrow \infty$.

1. Definition of $\varphi^{\lambda}$.

In order to justify that $\varphi^{\lambda}$ is well defined, we study separately the functions $m$ and $\ell$. We observe first that the function $\ell$ is negative, increasing and, according to (4.28), satisfies

$$
\ell(z) \sim_{\infty}-\int_{z}^{\infty} \frac{\lambda z^{\lambda} \alpha(u) d u}{u^{1+\lambda} S^{\prime}(u)} \sim_{\infty}-\frac{\lambda}{z S^{\prime}(z)} \rightarrow_{\infty} 0
$$

where the second equivalence comes from the following computation:

$$
-\int_{z}^{\infty} \frac{\lambda z^{\lambda} \alpha(u) d u}{u^{1+\lambda} S^{\prime}(u)}=\left[\frac{\lambda z^{\lambda}}{u^{1+\lambda} S^{\prime}(u)}\right]_{z}^{\infty}+\int_{z}^{\infty} \frac{\lambda(1+\lambda) z^{\lambda} d u}{u^{2+\lambda} S^{\prime}(u)}, \quad z>0 .
$$

We now turn to the study of $m$ and compute, for any $x>0$,

$$
m^{\prime}(x)=\frac{\{\lambda+1+x \alpha(x)\}}{\lambda S(x)^{3}} S^{\prime}(x) M(x), \quad \text { with } M: x \mapsto S(x)-\frac{2 x}{\lambda+1+x \alpha(x)} S^{\prime}(x) .
$$


Differentiating one more time, we obtain

$$
M^{\prime}(x)=\frac{x^{2} S^{\prime}(x)}{(\lambda+1+x \alpha(x))^{2}}\left[\frac{\lambda^{2}-1}{x^{2}}-\alpha(x)^{2}+2 \alpha^{\prime}(x)\right], \quad x>0 .
$$

Since $\lambda>1$ while $\alpha$ is non-negative, increasing and concave, the term in between brackets is decreasing. Furthermore $M^{\prime}(0)=\lambda-1>0$ and, since $x \alpha^{\prime}(x) \leq \alpha(x)$ for $x>0$, we get

$$
M^{\prime}(x) \leq \frac{S^{\prime}(x)}{(\lambda+1+x \alpha(x))^{2}}\left[\lambda^{2}-1-x^{2} \alpha(x)^{2}+2 x \alpha(x)\right] \rightarrow_{x \rightarrow \infty}-\infty .
$$

Thus $M$ is first increasing and then decreasing. Furthermore, estimate (4.28) implies that

$$
M(x) \sim_{\infty}\left[1-\frac{2 x \alpha(x)}{\lambda+1+x \alpha(x)}\right] S(x)=\frac{\lambda+1-x \alpha(x)}{\lambda+1+x \alpha(x)} S(x) \sim_{\infty}-S(x) .
$$

Since $M(0)=0$, we deduce that $m$ is first increasing and then decreasing. Then we have as $x \rightarrow 0, m(x) \sim \frac{1-\lambda}{\lambda x} \rightarrow-\infty$, and, using (4.28),

$$
m(x) \sim_{x \rightarrow \infty} \frac{x \alpha(x)-\lambda}{\lambda S(x)}>0, \quad \text { for sufficiently large } x .
$$

Since the function $\ell$ is negative, we deduce that, for any $z>0$, there is a unique point in $(0, \infty)$, denoted $\varphi^{\lambda}(z)$, such that $m \circ \varphi^{\lambda}(z)=\ell(z)$, and is the unique minimum of $x \mapsto g(x, z) / S(x)$ on $[0, \infty)$. This point is also the unique solution of

$$
g_{x}(x, z) S(x)-g(x, z) S^{\prime}(x)=0 .
$$

for any fixed $z$. The implicit functions theorem implies that $\varphi^{\lambda}$ is $C^{1}$ on $(0, \infty)$. We prove hereafter that $\varphi^{\lambda}(z)<z$, for any $z>0$, so that $\varphi^{\lambda}$ corresponds to the definition given in the statement of the lemma.

2. $\varphi^{\lambda}(z)<z$, for any $z>0$.

For any $z>0$, since $x \mapsto m(x)-\ell(z)$ is first negative and then positive, on $(0, \infty)$, the property $\varphi^{\lambda}(z)<z$ will be a direct consequence of the estimate $m(z)-\ell(z)>0$, that we prove now. First observe that the derivative of $h: z \mapsto[m(z)-\ell(z)] z^{-\lambda}$ is given by

$$
h^{\prime}(z)=\frac{1+z \alpha(z)}{z^{\lambda} S(z)^{3}} S^{\prime}(z) n(z), \quad z>0, \quad \text { with } \quad n: z \mapsto S(z)-\frac{2 z}{1+z \alpha(z)} S^{\prime}(z) .
$$

Hence $h^{\prime}$ has the same sign as $n$ and, differentiating one more time, we compute

$$
n^{\prime}(z)=S^{\prime}(z)\left[1-\frac{2+2 z \alpha(z)}{1+z \alpha(z)}+\frac{2 z\left(\alpha(z)+z \alpha^{\prime}(z)\right)}{|1+z \alpha(z)|^{2}}\right]=-\frac{1+z^{2} \alpha(z)^{2}-2 z^{2} \alpha^{\prime}(z)}{|1+z \alpha(z)|^{2}} S^{\prime}(z),
$$

for any $z>0$. Since $\alpha$ is concave and non-negative, we have $z \alpha^{\prime}(z) \leq \alpha(z)$ for $z>0$, and, plugging this estimate in the previous expression, we obtain

$$
n^{\prime}(z) \leq-\frac{|1-z \alpha(z)|^{2}}{|1+z \alpha(z)|^{2}} S^{\prime}(z) \leq 0, \quad z>0 .
$$


Hence, $n$ is non-increasing starting from $n(0)=0$, and therefore $h$ is also non-increasing on $(0, \infty)$. Furthermore, we know from (4.29) and (4.30) that $h(z)=[m(z)-\ell(z)] z^{-\lambda}>0$ for sufficiently large $z$, so that we have $m(z)-\ell(z)>0$, for any $z>0$.

3. $\varphi^{\lambda}$ is increasing and valued in $\left[0, y_{\lambda}\right]$.

Recall that $m \circ \varphi^{\lambda}=\ell$ and $\ell$ is increasing and negative. Since $m$ is also increasing when it is negative, we deduce that $\varphi^{\lambda}$ is increasing. Since after crossing zero, the function $m$ remains positive, $\varphi(z)$ must be smaller than the point where $m$ crosses zero, for any $z>0$. By definition of $m$, this point $y^{\lambda}$ is implicitly defined by $y^{\lambda} S^{\prime}\left(y^{\lambda}\right)=\lambda S\left(y^{\lambda}\right)$. Therefore $\varphi^{\lambda}(.) \leq y^{\lambda}$ and, since $\ell(z) \rightarrow_{z \rightarrow \infty} 0$, we even have $\varphi^{\lambda}(z) \rightarrow_{z \rightarrow \infty} y^{\lambda}$.

\section{Proof of Lemma 4.2}

Proof. We fix $\lambda>1$ and recall from estimate (4.17) in the proof of Theorem 4.1 that $\mathcal{L} g^{\lambda}$ is given by

$$
\mathcal{L} g^{\lambda}(x, z)=x^{\lambda-2}[x \alpha(x)+1-\lambda]\left(\frac{1}{z^{\lambda}}-\int_{z}^{\infty} \frac{S(x)}{S(u)} \frac{\lambda d u}{u^{1+\lambda}}\right)+2 x^{\lambda-1} S^{\prime}(x) \int_{z}^{\infty} \frac{\lambda d u}{S(u) u^{1+\lambda}},
$$

for any $0<x \leq z$. Since $S$ is increasing, we first observe that $\mathcal{L} g^{\lambda}(x,) \geq$.0 for any $x>0$ such that $x \alpha(x)+1-\lambda \geq 0$. Denoting by $x_{\lambda}$ the unique point of $\mathbb{R}^{+}$defined implicitly by

$$
x_{\lambda} \alpha\left(x_{\lambda}\right)=\lambda-1 \text {, }
$$

we deduce that $\mathcal{L} g^{\lambda}(x,) \geq$.0 for any $x \geq x_{\lambda}$.

It remains to treat the case where $x<x_{\lambda}$ and we compute

$$
\frac{\partial}{\partial z} \mathcal{L} g^{\lambda}(x, z)=\lambda \frac{x^{\lambda-2}}{z^{1+\lambda} S(z)}\left\{[\lambda-1-x \alpha(x)](S(z)-S(x))-2 x S^{\prime}(x)\right\}, \quad 0<x \leq z .
$$

For any fixed $x \in\left(0, x_{\lambda}\right)$, the previous expression in between brackets is increasing with respect to $z$, negative for $z=x$ and positive for $z$ large enough. Hence, for any $x \in\left(0, x_{\lambda}\right)$, $\mathcal{L} g(x,$.$) is first decreasing, then increasing and \mathcal{L} g(x, z)$ goes to 0 as $z$ goes to infinity. Denoting by $\gamma^{\lambda}$ the inverse of $\varphi^{\lambda}$, we deduce that

$$
\mathcal{L} g(x, z) \geq 0, \text { for any } z \leq \gamma^{\lambda}(x), \quad \text { if and only if } \quad \mathcal{L} g\left(x, \gamma^{\lambda}(x)\right) \geq 0,
$$

for any fixed $x \in\left(0, x^{\lambda}\right)$. Since $\varphi^{\lambda}$ and hence $\gamma^{\lambda}$ are increasing, it therefore only remains to verify that $\mathcal{L} g(., \gamma()) \geq$.0 on $\left(0, x_{\lambda}\right)$.

We recall from the proof of Lemma 4.1 that $\gamma^{\lambda}$ is defined implicitly by

$$
\int_{\gamma^{\lambda}(x)}^{\infty} \frac{\lambda\left[\gamma^{\lambda}(x)\right]^{\lambda} d u}{S(u) u^{1+\lambda}}=\frac{1}{S(x)}-\frac{x S^{\prime}(x)}{\lambda S(x)^{2}}, \quad 0<x<x_{\lambda} .
$$


For a given $x \in\left(0, x_{\lambda}\right)$, plugging this estimate into (4.32), we deduce

$$
\mathcal{L} g\left(x, \gamma^{\lambda}(x)\right)=(x \alpha(x)+1-\lambda) \frac{x^{\lambda-1} S^{\prime}(x)}{\lambda\left[\gamma^{\lambda}(x)\right]^{\lambda} S(x)}+\left(\frac{1}{S(x)}-\frac{x S^{\prime}(x)}{\lambda S^{2}(x)}\right) \frac{2 x^{\lambda-1} S^{\prime}(x)}{\left[\gamma^{\lambda}(x)\right]^{\lambda}},
$$

which after simplifications leads to

$\mathcal{L} g\left(x, \gamma^{\lambda}(x)\right)=\frac{(x \alpha(x)+1+\lambda) x^{\lambda-1} S^{\prime}(x)}{\lambda S(x)^{2}\left[\gamma^{\lambda}(x)\right]^{\lambda}} h(x), \quad$ with $h: x \mapsto S(x)-\frac{2 x}{x \alpha(x)+1+\lambda} S^{\prime}(x)$.

In order to get the sign of $\mathcal{L} g\left(., \gamma^{\lambda}().\right)$, we look for the sign of $h$ and compute

$$
\begin{aligned}
h^{\prime}(x) & =S^{\prime}(x)\left[1-\frac{2+2 x \alpha(x)}{1+\lambda+x \alpha(x)}+\frac{2 x\left(\alpha(x)+x \alpha^{\prime}(x)\right)}{|1+\lambda+x \alpha(x)|^{2}}\right] \\
& =\frac{S^{\prime}(x)}{|1+\lambda+x \alpha(x)|^{2}}\left[\lambda^{2}-1-x^{2} \alpha(x)^{2}+2 x^{2} \alpha^{\prime}(x)\right] \\
& \geq \frac{S^{\prime}(x)}{|1+\lambda+x \alpha(x)|^{2}}\left[\lambda^{2}-(1-x \alpha(x))^{2}\right], \quad x<x_{\lambda} .
\end{aligned}
$$

since $x \alpha^{\prime}(x) \leq \alpha(x)$ for $x>0$, due to the concavity of $\alpha$. By definition of $x_{\lambda}$, we deduce that $h$ is non-decreasing on $\left(0, x_{\lambda}\right)$. But $h(0)=0$ and therefore $\mathcal{L} g(., \gamma()) \geq$.0 on $\left(0, x_{\lambda}\right)$, which concludes the proof.

\section{Minimization of the relative quadratic error}

Let us now consider the case where $f: x \mapsto \frac{1}{2}(1-x)^{2}$. Therefore, we are computing the following value function

$$
V(x, z):=\frac{1}{2} \inf _{\theta \in \mathcal{T}} \mathbb{E}_{x, z}\left(1-\frac{X_{\theta}}{Z_{\tau}}\right)^{2}, \quad(x, z) \in \Delta .
$$

With such a criterion, the investor tries to minimize the expected value of the squared relative error between the value of the stopped process and the maximal value of the process up to $\tau$. In other words he wants to minimize the expectation of $\left[\left(Z_{\tau}-X_{\theta}\right) / Z_{\tau}\right]^{2}$, whereas in Section $4, \lambda=2$ would correspond to the minimization of $1-\left(X_{\theta} / Z_{\tau}\right)^{2}$, which is not as natural. In contrast with the previous optimal stopping problem (4.16), we prove that stopping immediately even for $x=z$ is not optimal in general. We exhibit the optimal liquidation strategy, which is numerically not that different from immediate selling.

\subsection{Construction of the solution}

From (2.11), we compute the corresponding reward function:

$$
g(x, z)=\frac{1}{2}\left(1-\frac{x}{z}\right)^{2}+x S(x) \int_{z}^{\infty}\left(1-\frac{x}{u}\right) \frac{d u}{u^{2} S(u)}, \quad(x, z) \in \boldsymbol{\Delta} .
$$


In view of Proposition 3.1, we would require $\mathcal{L} g(x, z) \geq 0$ in order for some $(x, z) \in \boldsymbol{\Delta}$ to be in the stopping region. Let us first compute

$$
\begin{aligned}
g_{x}(x, z) & =-\frac{1}{z}\left(1-\frac{x}{z}\right)+\left[S(x)+x S^{\prime}(x)\right] \int_{z}^{\infty} \frac{d u}{u^{2} S(u)}-\left[2 x S(x)+x^{2} S^{\prime}(x)\right] \int_{z}^{\infty} \frac{d u}{u^{3} S(u)}, \\
g_{x x}(x, z) & =\frac{1}{z^{2}}+[2+x \alpha(x)] S^{\prime}(x) \int_{z}^{\infty} \frac{d u}{u^{2} S(u)}-\left[2 S(x)+4 x S^{\prime}(x)+x^{2} \alpha(x) S^{\prime}(x)\right] \int_{z}^{\infty} \frac{d u}{u^{3} S(u)},
\end{aligned}
$$

for any $(x, z) \in \Delta$. Combining these estimates, we deduce

$$
\begin{aligned}
\mathcal{L} g(x, z)= & \frac{1}{z^{2}}[1+\alpha(x)(z-x)]+\left[2 S^{\prime}(x)-\alpha(x) S(x)\right] \int_{z}^{\infty} \frac{d u}{u^{2} S(u)} \\
& -\left[S(x)+2 x S^{\prime}(x)-x \alpha(x) S(x)\right] \int_{z}^{\infty} \frac{2 d u}{u^{3} S(u)}, \quad(x, z) \in \boldsymbol{\Delta} .
\end{aligned}
$$

In view of Theorem 3.1 (i), if $\mathcal{L} g \geq 0$ on $\boldsymbol{\Delta}$, then immediate stopping is optimal, $v=g$ and the problem is trivial. However, the next result gives sufficient conditions such that it is not the case. Consider the following condition:

$$
\alpha(0)^{2}-2 \alpha^{\prime}(0)<0
$$

Remark 5.1 Notice that (5.35) will be satisfied for an Ornstein-Uhlenbeck process as well as a CIR-Feller process with positive "mean", for which we respectively have $\alpha(x)=\alpha x$ and $\alpha(x)=\alpha \frac{x}{x+b}$ respectively, $\alpha$ and $b$ being positive constants. More generally, as soon as $\alpha(0)=0,(5.35)$ is satisfied. However, for a drifted Brownian motion or a degenerated CIR-Feller process with "mean" equal to 0, (5.35) does not hold true.

Proposition 5.1 Assume that (5.35) is satisfied. Then, $\mathcal{L} g(z, z)<0$ for $z$ small enough so that immediate stopping is not optimal for the problem of interest $V(z, z)$.

Proof. Using the asymptotic expansions from Proposition 5.4 in Section 5.4, we compute for $z$ close to 0 :

$$
\begin{aligned}
\mathcal{L} g(z, z)= & {\left[2 S^{\prime}(z)-\alpha(z) S(z)\right] \int_{z}^{\infty} \frac{d u}{u^{2} S(u)}-\left[S(z)+2 z S^{\prime}(z)-z \alpha(z) S(z)\right] \int_{z}^{\infty} \frac{2 d u}{u^{3} S(u)}+\frac{1}{z^{2}} } \\
= & \left(2+\alpha(0) z+O\left(z^{2}\right)\right)\left(\frac{1}{2 z^{2}}-\frac{\alpha(0)}{2 z}-\frac{\alpha(0)^{2}-2 \alpha^{\prime}(0)}{12} \ln z+o(\ln z)\right) \\
& \quad-\left(3 z+\frac{3}{2} \alpha(0) z^{2}+O\left(z^{3}\right)\right)\left(\frac{2}{3 z^{3}}-\frac{\alpha(0)}{2 z^{2}}+O\left(\frac{1}{z}\right)\right)+\frac{1}{z^{2}} \\
= & -\frac{\alpha(0)^{2}-2 \alpha^{\prime}(0)}{6} \ln z+o(\ln z) .
\end{aligned}
$$

Since $\ln z \rightarrow-\infty$ when $z \rightarrow 0$, we see that if (5.35) holds, then $\mathcal{L} g(z, z)<0$ for $z$ in a neighborhood of 0 , so that we have the result by continuity of $\mathcal{L} g$.

In particular, we deduce from Proposition 3.1 that immediate selling is not optimal for the problem $V(z, z)$ with $z$ small enough. 
Hence stopping immediately is not optimal in general and the optimal strategy shall be very different from the one in the power utility case. Since we do not have $\mathcal{L} g \geq 0$ on the entire space $\boldsymbol{\Delta}$ but we can exercise only in that region, we first need to study the set

$$
\Gamma^{+}:=\{(x, z) \in \boldsymbol{\Delta}, \mathcal{L} g(x, z) \geq 0\}
$$

and we define similarly:

$$
\Gamma^{-} \quad:=\{(x, z) \in \boldsymbol{\Delta}, \mathcal{L} g(x, z) \leq 0\}
$$

In fact, observe that (5.34) rewrites as:

$$
\begin{aligned}
\mathcal{L} g(x, z)= & \alpha(x) \frac{z-x}{z^{2}}+\left[2 S^{\prime}(x)-\alpha(x) S(x)\right] \int_{z}^{\infty}\left(1-\frac{2 x}{u}\right) \frac{d u}{u^{2} S(u)} \\
& +\left(\frac{1}{z^{2}}-S(x) \int_{z}^{\infty} \frac{2 d u}{u^{3} S(u)}\right), \quad(x, z) \in \boldsymbol{\Delta} .
\end{aligned}
$$

By Remark 2.3, we have $2 S^{\prime}-\alpha S-2 \geq 0$ and therefore each of the three terms above are positive if $z \geq 2 x$, and so

$$
\mathcal{L} g(x, z)>0 \quad \text { for } \quad z \geq 2 x \quad \text { and } \quad(x, z) \in \boldsymbol{\Delta}
$$

which implies that $\{(x, z) \in \boldsymbol{\Delta}, z \geq 2 x\} \subset \operatorname{Int}\left(\Gamma^{+}\right)$.

Moreover we have the following result, which proof is given in Section 5.4 below.

Lemma 5.1 For any $x>0$, there exists $\delta_{x} \in(x, 2 x)$ such that $\mathcal{L} g(x,$.$) is increasing on$ $\left[x, \delta_{x}\right)$ and decreasing on $\left(\delta_{x}, 2 x\right]$.

In view of (5.39), we can define the following function on $\mathbb{R}_{+} \backslash\{0\}$ :

$$
\Gamma(x):=\inf \{z \geq x, \mathcal{L} g(x, z) \geq 0\}
$$

Lemma 5.1 and (5.39) imply that, if $z>\Gamma(x)$, then $\mathcal{L} g(x, z)>0$, while if $z \in(x, \Gamma(x))$, then $\mathcal{L} g(x, z)<0$. We also deduce that $\Gamma(x)>x$ implies $\mathcal{L} g(x, \Gamma(x))=0$. Notice that $\Gamma$ is continuous, and, from (5.39), we also know that $\Gamma(x)<2 x$.

The next result provides the main properties of $\Gamma$ : it is increasing and equal to the Identity function for sufficiently large $x$. Again the proof is postponed to Section 5.4.

Proposition 5.2 We have the two following properties:

(i) $\Gamma$ is increasing on $(0,+\infty)$;

(ii) Denoting $\Gamma^{\infty}:=\sup \{x \geq 0 ; \Gamma(x)>x\}$, we get $\Gamma^{\infty}<\infty$.

Notice that $\Gamma^{+} \neq \Delta$ implies directly $\Gamma^{\infty}>0$. 
Now that we have a better understanding of the set $\Gamma^{+}$, we expect to have a stopping region of the form $\{(x, z) \in \Delta ; z \geq \gamma(x)\}$, and our objective is then to find functions $v$ and $\gamma$, satisfying the following free-boundary problem:

$$
\begin{aligned}
\mathcal{L} v(x, z) & =0 \quad \text { for } x \leq z<\gamma(x) \quad \text { and } \quad(x, z) \in \boldsymbol{\Delta} \\
v(x, z) & =g(x, z) \quad \text { and } \quad \mathcal{L} g(x, z) \geq 0 \quad \text { for } z \geq \gamma(x) \quad \text { and } \quad(x, z) \in \boldsymbol{\Delta} \\
v(0, z) & =\frac{1}{2} \quad \text { for } z>0 \\
v_{z}(z, z) & =0 \quad \text { for } z>0
\end{aligned}
$$

In order to allow for the application of Itô's formula, the verification step requires a value function which is $C^{1,0}$ and piecewise $C^{2,1}$ with respect to $(x, z)$. Therefore, as in Section 4 , we complement the above system by the continuity and the smooth fit conditions

$$
v(x, \gamma(x))=g(x, \gamma(x)) \quad \text { and } \quad v_{x}(x, \gamma(x))=g_{x}(x, \gamma(x)), \quad \text { for } x>0
$$

The stopping region $\mathcal{S}$ will then be defined as:

$$
\mathcal{S}:=\{(x, z) \in \Delta ; z \geq \gamma(x)\} \cup\{(0, z) ; z>0\}
$$

First by (5.41), on the continuation region, $v$ is of the form:

$$
v(x, z)=A(z)+B(z) S(x), \quad(x, z) \in \boldsymbol{\Delta} \backslash \mathcal{S} .
$$

Then, on the interval where $\gamma$ is one-to-one, the continuity and smoothfit conditions (5.45) imply that

$$
v(x, z)=g\left(\gamma^{-1}(z), z\right)+\frac{g_{x}\left(\gamma^{-1}(z), z\right)}{S^{\prime} \circ \gamma^{-1}(z)}\left[S(x)-S \circ \gamma^{-1}(z)\right], \quad(x, z) \in \boldsymbol{\Delta} \backslash \mathcal{S} .
$$

Finally, the Neumann condition (5.44), implies that we expect the boundary $\gamma$ to satisfy on its domain of definition the following ODE:

$$
\gamma^{\prime}(x)=\frac{\gamma(x)^{2} \mathcal{L} g(x, \gamma(x))}{\left(\frac{2 x}{\gamma(x)}-1\right)\left(1-\frac{S(x)}{S \circ \gamma(x)}\right)} .
$$

As in [5], there is no a priori initial condition for this ODE. In the sequel, we take this ODE (with no initial condition) as a starting point to construct the boundary $\gamma$. Notice that this ODE has infinitely many solutions, as the Cauchy-Lipschitz condition is locally satisfied whenever (5.47) is complemented with the condition $\gamma\left(x_{0}\right)=z_{0}$ for any $0<x_{0}<z_{0}$ and $z_{0} \neq 2 x_{0}$. We will follow the ideas of [5], however in our case, (5.47) is not well-defined for $\gamma(x)=2 x$, so that our framework requires to be more cautious. Notice also that we encounter here a similar feature as in Peskir [8]. The following result selects an appropriate solution of (5.47), and its proof is given in Section 5.5. 
Proposition 5.3 Let $\operatorname{Int}\left(\Gamma^{-}\right)$be non empty. Then, there exists an increasing continuous function $\gamma$ defined on $\mathbb{R}_{+}$with graph $\{(x, \gamma(x)): x>0\} \subset \Delta$, such that:

(i) On the set $\{x>0: \gamma(x)>x\}, \gamma$ is a $C^{1}$ solution of the ODE (5.47),

(ii) $\{(x, \gamma(x)): x>0\} \subset \Gamma^{+}$, and $\{(x, \gamma(x)): x>0$ and $\gamma(x)>x\} \subset \operatorname{Int}\left(\Gamma^{+}\right)$,

(iii) $\gamma(x)=x$ for all $x \geq \Gamma^{\infty}$.

Since $\gamma$ is increasing, we can define:

$$
\varphi:=\gamma^{-1}
$$

Now that we have constructed the free-boundary $\varphi$, we are able to state the following result.

Theorem 5.1 Let Int $\left(\Gamma^{-}\right)$be non empty, $\gamma$ be given by Proposition 5.3 and $\varphi$ be defined by (5.48). Then the value function $V$ solution of problem (5.33) is given, for $(x, z) \in \boldsymbol{\Delta}$, by:

$$
V(x, z):=\left\{\begin{array}{ll}
g(x, z), & \text { if } x \leq \varphi(z) \\
g(\varphi(z), z)+g_{x}(\varphi(z), z) \frac{S(x)-S \circ \varphi(z)}{S^{\prime} \circ \varphi(z)}, & \text { if } x>\varphi(z)
\end{array} .\right.
$$

Moreover, the smallest optimal stopping time associated to (5.33) is given by $\theta^{*}:=\inf \{t \geq$ $\left.0, \quad X_{t} \leq \varphi\left(Z_{t}\right)\right\}$.

Proof. Let $v$ be defined by (5.49) and recall that $\mathcal{S}$ is defined by (5.46). The result follows from verifying that all the assumptions of Theorem 3.1 (ii) and (iii) are satisfied.

1. Regularity of $v$.

We know from Proposition 5.3 that $\gamma$ and therefore $\varphi$ are continuous and hence $v$ is continuous on $\boldsymbol{\Delta}$ by construction. Furthermore, by Proposition 5.3 (i) and (ii) together with the dynamics of the $\operatorname{ODE}(5.47), \gamma$ is a $C^{1}$ function with positive derivative on the set $\{x>0 ; \gamma(x)>x\}$. Therefore $\varphi$ is $C^{1}$ as well on $\{z>0 ; \varphi(z)<z\}$ so that it is immediate that $v$ is $C^{0}$ and piecewise $C^{2,1}$ w.r.t. $(x, z)$. Furthermore, since $\Gamma^{\infty}<\infty$ by Proposition $5.2, \boldsymbol{\Delta} \backslash \mathcal{S}$ is bounded. Since $v$ is continuous and $g \geq 0, v$ is bounded from below.

2. Dynamics of $v$.

By definition, we have $\mathcal{L} v=0$ on $\boldsymbol{\Delta} \backslash \mathcal{S}$. By Proposition 5.3 (ii), $\mathcal{L} g(x, \gamma(x)) \geq 0$ for $x>0$, and we deduce from Lemma 5.1 and (5.39) that $\mathcal{L} g(x, z) \geq 0$ for any $(x, z) \in \boldsymbol{\Delta}$ such that $z \geq \gamma(x)$. Hence, (5.39) ensures that $\mathcal{L} g \geq 0$ on $\mathcal{S}$.

It remains to prove that $v_{z}(z, z)=0$ for $z>0$. We fix $z>0$. If $\varphi(z) \geq z$, since $g_{z}(z, z)=0$, we have $v_{z}(z, z)=0$ as well. Suppose now that $\varphi(z)<z$. Then, by Proposition 5.3 (i), $\gamma$ satisfies (5.47) in a neighborhood of $\varphi(z)$, and by Proposition 5.3 (ii), $\mathcal{L} g(\varphi(z), z)>0$, which implies $\gamma^{\prime} \circ \varphi(z)>0$, so that:

$$
\varphi^{\prime}(z) \mathcal{L} g(\varphi(z), z)=\frac{1}{z^{2}}\left(\frac{2 \varphi(z)}{z}-1\right)\left(1-\frac{S \circ \varphi(z)}{S(z)}\right) .
$$


We then compute from the definitions of $v$ and $g$ that

$$
\begin{aligned}
v_{z}(z, z) & =g_{z}(\varphi(z), z)+g_{x z} \frac{S(z)-S \circ \varphi(z)}{S^{\prime} \circ \varphi(z)}+\varphi^{\prime}(z) \mathcal{L} g(\varphi(z), z) \frac{S(z)-S \circ \varphi(z)}{S^{\prime} \circ \varphi(z)} \\
& =\left[\frac{1}{z^{2}}\left(1-\frac{2 \varphi(z)}{z}\right)\left(1-\frac{S \circ \varphi(z)}{S(z)}\right)+\varphi^{\prime}(z) \mathcal{L} g(\varphi(z), z)\right] \frac{S(z)-S \circ \varphi(z)}{S^{\prime} \circ \varphi(z)}=0 .
\end{aligned}
$$

\section{Comparing $v$ and $g$.}

Finally, the fact that $v \leq g$ on $\boldsymbol{\Delta}$ and $v<g$ on $\boldsymbol{\Delta} \backslash \mathcal{S}$ follows from similar arguments as in the proof of Proposition 6.2 in [5] but the demonstration is simpler in our context since $\Gamma^{\infty}<\infty$. For the sake of completeness, we detail this proof. For $(x, z) \in \boldsymbol{\Delta}$ such that $x>\varphi(z)$, we compute

$$
v(x, z)-g(x, z)=g(\varphi(z), z)+g_{x}(\varphi(z), z) \frac{S(x)-S \circ \varphi(z)}{S^{\prime} \circ \varphi(z)}-g(x, z),
$$

and, differentiating twice w.r.t. $x$ and using (5.45), we verify that

$$
v_{x}(x, z)-g_{x}(x, z)=-S^{\prime}(x) \int_{\varphi(z)}^{x} \frac{\mathcal{L} g(u, z)}{S^{\prime}(u)} d u .
$$

Therefore, from Lemma 5.1 and Proposition 5.2 (i), for any fixed $z$, the function $x \mapsto$ $(v-g)(x, z)$ is either decreasing on $[\varphi(z), z]$, or decreasing on $[\varphi(z), \delta)$ and then increasing on $(\delta, z]$ for a given $\delta \in(\varphi(z), z)$. For any $z>0$, since $v(\varphi(z), z)=g(\varphi(z), z)$, we only need to prove that $n(z):=v(z, z)-g(z, z)<0$ if $\varphi(z)<z$.

Since $v_{z}(z, z)=g_{z}(z, z)=0$ for $z>0$, we compute:

$$
n^{\prime}(z)=v_{x}(z, z)-g_{x}(z, z)=-S^{\prime}(z) \int_{\varphi(z)}^{z} \frac{\mathcal{L} g(u, z)}{S^{\prime}(u)} d u, \quad z>0 .
$$

We assume the existence of a fixed $z<\Gamma^{\infty}$ such that $n(z) \geq 0$ and $\varphi(z)<z$ and work to-

wards a contradiction. We first observe that necessarily $n^{\prime}(z)>0$. If not, $\int_{\varphi(z)}^{z} \frac{\mathcal{L} g(u, z)}{S^{\prime}(u)} d u \geq 0$ implies that $\int_{\varphi(z)}^{x} \frac{\mathcal{L} g(u, z)}{S^{\prime}(u)} d u>0$ for any $x \in(\varphi(z), z)$, and (5.50) combined with $v(\varphi(z), z)=$ $g(\varphi(z), z)$ leads to $n(z)<0$ which is impossible. Since $n$ is continuous, this implies that $n$ is increasing on any connected subset of $\left\{z^{\prime} \geq z, \varphi\left(z^{\prime}\right)<z^{\prime}\right\}$. Defining $a:=\inf \left\{z^{\prime}>\right.$ $\left.z ; \varphi\left(z^{\prime}\right)=z^{\prime}\right\} \leq \Gamma^{\infty}<\infty$, we get $n(a)=v(a, a)-g(a, a)>0$, which contradicts the definition of $v$.

Therefore, $n(z)<0$ for any $z>0$ such that $\varphi(z)<z$ and we deduce that $v \leq g$ on $\boldsymbol{\Delta}$ and $v<g$ on $\boldsymbol{\Delta} \backslash \mathcal{S}$.

\subsection{Properties of the optimal liquidation strategy}

Theorem 5.1 and Proposition 5.1 indicate that, at least for processes satisfying (5.35), such as the Ornstein-Uhlenbeck process or the CIR-Feller process, the diagonal $\{(x, x) ; x>0\}$ 
is not included in the stopping region $\mathcal{S}$. In other words, it is not always optimal to stop immediately, even when starting from points such that $x=z$. Therefore, the form of the solution and the nature of the optimal strategy to apply in order to be as close as possible to the maximum using this criterion is very different from the ones obtained in Section 4 or in $[9]$.

The Ornstein-Uhlenbeck process as well as the CIR-Feller process are two examples for which the coefficient $\alpha$ satisfies Conditions (2.12) and $\operatorname{Int}\left(\Gamma^{-}\right) \neq \emptyset$. Indeed we have $\alpha(x)=\alpha x$ and $\alpha(x)=\alpha \frac{x}{x+b}$ respectively, where $\alpha$ and $b$ are two positive constants. Therefore, Condition (5.35) is satisfied, ensuring that $\operatorname{Int}\left(\Gamma^{-}\right) \neq \emptyset$ by Proposition 5.1. Hence, Theorem 5.1 can be applied. Figure 2 represents the boundary $\varphi$ for those two processes, with $\alpha=1$ for the OU process and $(\alpha, b)=(0.1,0.1)$ for the CIR process. We observe that the continuation region is in fact pretty small since the free boundary is very close to the diagonal axis. Therefore, even if immediate stopping is not optimal, an investor should not wait long until the process $\left(X, X^{*}\right)$ enters the stopping region.

Free Boundary of OU

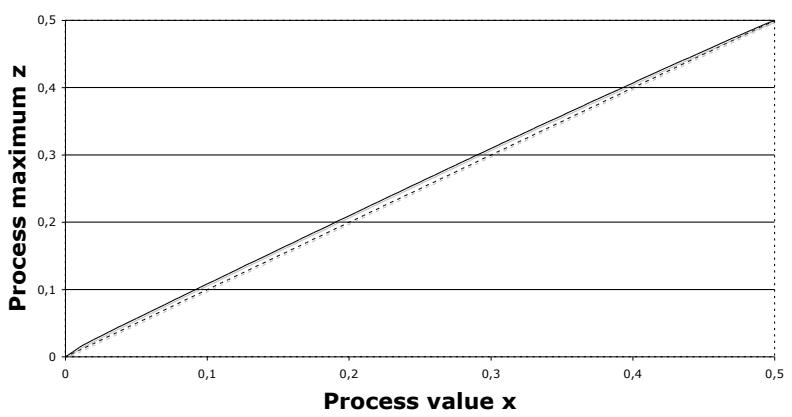

Free Boundary of CIR

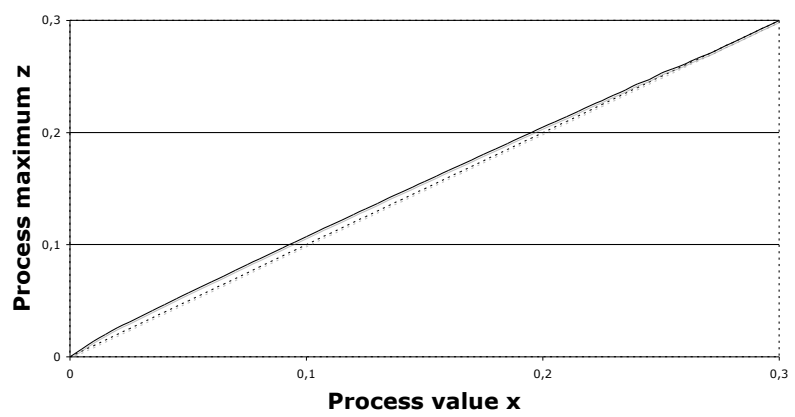

Figure 2: Optimal frontier for an $\mathrm{OU}(\alpha=1)$ and a $\mathrm{CIR}(\alpha=0.1, b=0.1)$

Remark 5.2 Similarly to Proposition 7.3 of [5], an homogeneity result can be derived for the OU process, so that the free boundary for any $\alpha>0$ can be deduced by a change of scale from the one for $\alpha=1$.

The Brownian motion with negative drift is another example for which $\alpha$ satisfies Condition (2.12). However, since $\alpha(x)=\alpha>0$ is constant, Condition (5.35) does not hold. Although we did not verify it, numerical computations suggest that $\mathcal{L} g \geq 0$ on $\boldsymbol{\Delta}$.

Finally, we can also consider the case of a Brownian motion. In this case, $\alpha(x)=0$, so that $\alpha$ does not satisfy Condition (2.12). However, for any $(x, z) \in \boldsymbol{\Delta}$, we can compute from 
(5.34) that $\mathcal{L} g(x, z)=2 \frac{z-x}{z^{3}} \geq 0$ on $\boldsymbol{\Delta}$. Since the proofs of Theorem 3.1 and Remark 3.2 do not require Condition (2.12), we deduce that immediate stopping is always optimal.

Remark 5.3 Let $\alpha$ be associated to an Ornstein-Uhlenbeck or a CIR process and hence be parametrized by a possibly bi-dimensional parameter set $a$. Since the parameter set $a$ may be badly estimated, let consider a sequence of parameter set $\left(a_{n}\right)$ converging to $a$ and denote by $\left(\alpha_{n}\right)$ the corresponding sequence of functions. Then, $S_{n}, g_{n}$ and all their derivatives converge respectively to $S, g$ and their derivatives in the sense of the uniform norm on the compact sets. Moreover, $\Gamma_{n}$ converges to $\Gamma$ in the same sense so that for $n$ sufficiently large, $\operatorname{Int}\left(\Gamma_{n}^{-}\right) \neq \emptyset$. ODE (5.47) also depends continuously on $a_{n}$, so that $z_{n}^{*}\left(x_{0}\right)$ defined by (5.63) converges to $z^{*}\left(x_{0}\right)$, and $\gamma_{n}$ given by Proposition 5.3 converges pointwise to $\gamma$. Since $\gamma_{n}$ is increasing for any $n$ and $\gamma$ is continuous, Dini's theorem implies that the convergence is uniform on any compact set of $\mathbb{R}_{+} \backslash\{0\}$. Let us prove that $\varphi_{n}$ converges to $\varphi$ in the same sense. Let $y>0$ be fixed, we define $x_{n}:=\varphi_{n}(y)$ and $x:=\varphi(y)$. We shall prove that $x_{n} \rightarrow x$. Indeed, since $\varphi_{n}(y) \in\left[\frac{y}{2}, y\right]$ for any $n,\left\{x_{n} ; n \in \mathbb{N}\right\}$ is relatively compact in $\mathbb{R}_{+} \backslash\{0\}$. Now let $x^{\prime}$ be the limit of a subsequence of $\left(x_{n}\right)$. For notational reasons, let us write $x_{n} \rightarrow x^{\prime}$, forgetting that it is a subsequence. Since $\left(\gamma_{n}\right)$ converges uniformly on compact sets of $\mathbb{R}_{+} \backslash\{0\}, \gamma_{n}\left(x_{n}\right) \rightarrow \gamma\left(x^{\prime}\right)$. Recalling that $\gamma_{n}\left(x_{n}\right)=y$ for any $n$, we get $\gamma\left(x^{\prime}\right)=y$ and therefore $x^{\prime}=x$. In consequence, $x_{n} \rightarrow x$, or in other words, $\left(\varphi_{n}\right)$ converges pointwise to $\varphi$ on $\mathbb{R}_{+} \backslash\{0\}$. Noticing that $\varphi_{n}(0)=0$ for any $n$ and $\varphi(0)=0$ and using again Dini's theorem, we see that $\left(\varphi_{n}\right)$ converges to $\varphi$ uniformly on the compact sets of $\mathbb{R}_{+}$. This finally implies that $\left(V_{n}\right)$ converges pointwise to $V$. As a consequence, if one makes a small mistake estimating the parameters of the model, the induced mistake on the free boundary as well as the mistake on the value function will be small as well.

\subsection{Generalization}

As in Section 4, we may also consider, for any $\lambda>0$, the following extension of the previous problem:

$$
V_{\lambda}(x, z):=\frac{1}{\lambda} \inf _{\theta \in \mathcal{T}} \mathbb{E}_{x, z}\left(1-\frac{X_{\theta}}{Z_{\tau}}\right)^{\lambda}, \quad(x, z) \in \Delta .
$$

In that case, (2.10) rewrites

$$
g_{\lambda}(x, z)=\frac{1}{\lambda}\left(1-\frac{x}{z}\right)^{\lambda}+x S(x) \int_{z}^{\infty}\left(1-\frac{x}{u}\right)^{\lambda-1} \frac{d u}{u^{2} S(u)} d u, \quad(x, z) \in \boldsymbol{\Delta}, \quad \lambda>0 .
$$

If $\lambda=2, \mathcal{L} g_{2}$ is given by (5.34). If $\lambda=1$, the stopping problem has already been solved in Section 4 and $\mathcal{L} g_{1}$ is given by (4.18). For any $\lambda>0$ such that $\lambda \notin\{1,2\}$, we compute :

$$
\begin{aligned}
\mathcal{L} g_{\lambda}(x, z)= & (\lambda-1)\left\{\frac{1}{z^{2}}\left(1-\frac{x}{z}\right)^{\lambda-2}-\int_{z}^{\infty} \frac{S(x)}{S(u)}\left[\frac{2}{u^{3}}\left(1-\frac{x}{u}\right)^{\lambda-2}-\frac{(\lambda-2) x}{u^{4}}\left(1-\frac{x}{u}\right)^{\lambda-3}\right] d u\right\} \\
& +\left[2 S^{\prime}(x)-\alpha(x) S(x)\right] \int_{z}^{\infty}\left(1-\frac{x}{u}\right)^{\lambda-2}(u-\lambda x) \frac{d u}{u^{3} S(u)}+\alpha(x) \frac{(z-x)^{\lambda-1}}{z^{\lambda}},(5.53)
\end{aligned}
$$


for $0 \leq x<z$. In this case, the sign of $\mathcal{L} g_{\lambda}$ is hardly identifiable analytically, and we shall restrict our analysis to simple remarks and guesses on the solution of the problem (5.51).

Noticing that $\int_{z}^{\infty}\left(1-\frac{x}{u}\right)^{\lambda-2} \frac{2}{u^{3}}-(\lambda-2)\left(1-\frac{x}{u}\right)^{\lambda-3} \frac{x}{u^{4}} d u=\frac{1}{z^{2}}\left(1-\frac{x}{z}\right)^{\lambda-2}$ for $0<x<z$, we deduce from (4.18), (5.39) and (5.53) that

$$
\mathcal{L} g^{\lambda}(x, z) \geq 0, \quad \text { for } z \geq \lambda x>0 \quad \text { and } 1 \leq \lambda \leq 2 .
$$

Therefore, for $1 \leq \lambda \leq 2$, we expect to obtain as for $\lambda=2$ a free boundary $\gamma_{\lambda}$ in between the axis $\{(x, x) ; x>0\}$ and $\{(x, \lambda x) ; x>0\}$. We verify easily as in Proposition (4.1) that $\lambda \mapsto V_{\lambda}$ is continuous and, as expected, we observe a disappearance of the free boundary $\gamma_{\lambda}$ for $\lambda=1$.

On the other hand, in the case where $\alpha(0)=0$, for $\lambda<1$, we observe that $\mathcal{L} g(x, z)<0$ for $x$ small enough and $z$ large enough. Indeed, recalling from (4.28) that $S^{\prime}(z) \sim \alpha(z) S(z)$ when $z \rightarrow \infty$, an integration by parts leads to $\int_{z}^{\infty} \frac{d u}{u^{2} S(u)} \sim_{z \rightarrow \infty} \frac{1}{z^{2} S^{\prime}(z)}$. Assuming moreover that $\alpha(0)=0$ and plugging this estimate in (5.53), we get

$$
\mathcal{L} g(0, z) \quad \sim_{z \rightarrow \infty} \frac{\lambda-1}{z^{2}}<0, \quad \text { for any } \lambda<1 .
$$

In view of Proposition 3.1, this implies that the stopping region cannot have the same form as the one in the quadratic case $\lambda=2$. It even suggests that the nature of the stopping region could be similar to the one of Section 4.2.

\subsection{Proofs of Lemma 5.1 and Proposition 5.2}

This section is dedicated to the proofs of Lemma 5.1 and Proposition 5.2, but we first state the asymptotic expansions used in Proposition 5.1.

Proposition 5.4 As $z \rightarrow 0$, we have the following expansions:

$$
\begin{aligned}
S^{\prime}(z) & =1+\alpha(0) z+\left(\alpha^{\prime}(0)+\alpha(0)^{2}\right) \frac{z^{2}}{2}+o\left(z^{2}\right) ; \\
S(z) & =z+\alpha(0) \frac{z^{2}}{2}+\left(\alpha^{\prime}(0)+\alpha(0)^{2}\right) \frac{z^{3}}{6}+o\left(z^{3}\right) ; \\
\alpha(z) S(z) & =z \alpha(0)+\frac{z^{2}}{2}\left(\alpha(0)^{2}+2 \alpha^{\prime}(0)\right)+\frac{z^{3}}{6}\left(\alpha(0)^{3}+4 \alpha(0) \alpha^{\prime}(0)+3 \alpha^{\prime \prime}(0)\right)+o\left(z^{3}\right) ; \\
\int_{z}^{\infty} \frac{d u}{u^{2} S(u)} & =\frac{1}{2 z^{2}}-\frac{\alpha(0)}{2 z}-\frac{\alpha(0)^{2}-2 \alpha^{\prime}(0)}{12} \ln (z)+o(\ln (z)) ; \\
\int_{z}^{\infty} \frac{2 d u}{u^{3} S(u)} & =\frac{2}{3 z^{3}}-\frac{\alpha(0)}{2 z^{2}}+\frac{\alpha(0)^{2}-2 \alpha^{\prime}(0)}{6 z}+o\left(\frac{1}{z}\right) .
\end{aligned}
$$

Proof. As $z \rightarrow 0$, we directly compute the expansion:

$$
S^{\prime}(z)=S^{\prime}(0)+z S^{\prime \prime}(0)+\frac{z^{2}}{2} S^{(3)}(0)+o\left(z^{2}\right)=1+\alpha(0) z+\left(\alpha^{\prime}(0)+\alpha(0)^{2}\right) \frac{z^{2}}{2}+o\left(z^{2}\right) .
$$


The exact same reasoning also leads to

$$
\begin{aligned}
S(z) & =z+\frac{\alpha(0)}{2} z^{2}+\frac{\alpha^{\prime}(0)+\alpha(0)^{2}}{6} z^{3}+o\left(z^{3}\right) ; \\
\alpha(z) S(z) & =z \alpha(0)+\frac{z^{2}}{2}\left(\alpha(0)^{2}+2 \alpha^{\prime}(0)\right)+\frac{z^{3}}{6}\left(\alpha(0)^{3}+4 \alpha(0) \alpha^{\prime}(0)+3 \alpha^{\prime \prime}(0)\right)+o\left(z^{3}\right) .
\end{aligned}
$$

Using one of the previous estimates, we get

$$
\begin{aligned}
\int_{z}^{\infty} \frac{d u}{u^{2} S(u)} & =\int_{z}^{\infty} \frac{d u}{u^{3}\left(1+\frac{\alpha(0)}{2} u+\frac{\alpha^{\prime}(0)+\alpha(0)^{2}}{6} u^{2}+o\left(u^{2}\right)\right)} \\
& =\int_{z}^{\infty}\left(1-\frac{\alpha(0)}{2} u-\frac{\alpha^{\prime}(0)+\alpha(0)^{2}}{6} u^{2}+\left(\frac{\alpha(0) u}{2}\right)^{2}+o\left(u^{2}\right)\right) \frac{d u}{u^{3}} \\
& =\int_{z}^{\infty}\left(\frac{1}{u^{3}}-\frac{\alpha(0)}{2 u^{2}}+\frac{\alpha(0)^{2}-2 \alpha^{\prime}(0)}{12 u}+o\left(\frac{1}{u}\right)\right) d u \\
& =\frac{1}{2 z^{2}}-\frac{\alpha(0)}{2 z}-\frac{\alpha(0)^{2}-2 \alpha^{\prime}(0)}{12} \ln (z)+o(\ln (z)),
\end{aligned}
$$

which is justified since all the non-zero terms go to infinity when $z \rightarrow 0$. Similarly, we compute

$$
\begin{aligned}
\int_{z}^{\infty} \frac{2 d u}{u^{3} S(u)} & =\int_{z}^{\infty}\left(\frac{2}{u^{4}}-\frac{\alpha(0)}{u^{3}}+\frac{\alpha(0)^{2}-2 \alpha^{\prime}(0)}{6 u^{2}}+o\left(\frac{1}{u^{2}}\right)\right) d u \\
& =\frac{2}{3 z^{3}}-\frac{\alpha(0)}{2 z^{2}}+\frac{\alpha(0)^{2}-2 \alpha^{\prime}(0)}{6 z}+o\left(\frac{1}{z}\right) .
\end{aligned}
$$

Proof of Lemma 5.1 Differentiating (5.34) w.r.t. $z$, we compute

$$
\begin{aligned}
\frac{\partial}{\partial z} \mathcal{L} g(x, z) & =-\frac{2 S^{\prime}(x)-\alpha(x) S(x)}{z^{2} S(z)}+\frac{[2-2 x \alpha(x)] S(x)+4 x S^{\prime}(x)}{z^{3} S(z)}-\frac{2-2 x \alpha(x)}{z^{3}}-\frac{\alpha(x)}{z^{2}} \\
& =[(2 x-z) \alpha(x)-2] \frac{S(z)-S(x)}{z^{3} S(z)}+(2 x-z) \frac{2 S^{\prime}(x)}{z^{3} S(z)}, \quad(x, z) \in \boldsymbol{\Delta} .
\end{aligned}
$$

Let us introduce $x_{\alpha}$ as the unique solution of:

$$
x_{\alpha} \alpha\left(x_{\alpha}\right)=2 .
$$

If $x \leq x_{\alpha}$, then $z \mapsto(2 x-z) \alpha(x)-2$ is negative on $[x, 2 x)$, whereas if $x>x_{\alpha}$, then there exists $z_{x} \in(x, 2 x)$ such that $z \mapsto(2 x-z) \alpha(x)-2$ will be positive on $\left(x, z_{x}\right)$, zero at $z_{x}$ and negative on $\left(z_{x}, 2 x\right)$.

Let $x$ be fixed and let us introduce

$$
F \quad: \quad z \mapsto S(z)-S(x)+\frac{2 S^{\prime}(x)(2 x-z)}{(2 x-z) \alpha(x)-2},
$$


which is well defined and continuous on $[x, 2 x]$ if $x<x_{\alpha}$, on $[x, 2 x)$ if $x=x_{\alpha}$ and on $[x, 2 x] \backslash\left\{z_{x}\right\}$ if $x>x_{\alpha}$. Furthermore $F$ is increasing, since we compute on the domain of definition of $F$ :

$$
F^{\prime}(z)=S^{\prime}(z)+\frac{4 S^{\prime}(x)}{((2 x-z) \alpha(x)-2)^{2}}>0 .
$$

We consider first the case where $x \leq x_{\alpha}$. Then $F$ and $\frac{\partial}{\partial z} \mathcal{L} g(x,$.$) have opposite signs on$ $[x, 2 x)$. Since $F$ is increasing, $F(x)<0$ while $F(2 x)=S(2 x)-S(x)>0, \mathcal{L} g(x,$.$) is$ increasing on $\left[x, \delta_{x}\right)$ and decreasing on $\left(\delta_{x}, 2 x\right]$, for a certain $\delta_{x} \in(x, 2 x)$.

We now turn to the case where $x>x_{\alpha}$. Then $F$ and $\frac{\partial}{\partial z} \mathcal{L} g(x,$.$) have the same sign on$ $\left[x, z_{x}\right)$ and opposite signs on $\left(z_{x}, 2 x\right]$. Since $F$ is increasing, $F(x)>0, F\left(z_{x}^{+}\right)=-\infty$ and $F(2 x)>0$, we see that again $\mathcal{L} g(x,$.$) is increasing on \left[x, \delta_{x}\right)$ and decreasing on $\left(\delta_{x}, 2 x\right]$, for a certain $\delta_{x} \in\left(z_{x}, 2 x\right) \subset(x, 2 x)$.

Proof of Proposition 5.2 We prove the two assertions separately.

(i) $\Gamma$ is increasing on $(0,+\infty)$

We fix $x>0$ such that $\Gamma(x)>x$. Then $\mathcal{L} g(., \Gamma())=$.0 in a neighborhood of $x$, and using the implicit functions theorem, $\Gamma$ is $C^{1}$ in a neighborhood of $x$ and we have:

$$
\Gamma^{\prime}(x) \frac{\partial}{\partial z} \mathcal{L} g(x, \Gamma(x))+\frac{\partial}{\partial x} \mathcal{L} g(x, \Gamma(x))=0 .
$$

We will prove that $\Gamma^{\prime}(x)>0$. Denoting $m:=2 S^{\prime}-\alpha S$ which is increasing and positive, we get combining $\mathcal{L} g(x, \Gamma(x))=0$ and (5.38):

$$
m(x) \int_{\Gamma(x)}^{\infty} \frac{u-2 x}{u^{3} S(u)} d u=S(x) \int_{\Gamma(x)}^{\infty} \frac{2 d u}{u^{3} S(u)}-\frac{1+\alpha(x)(\Gamma(x)-x)}{\Gamma(x)^{2}} \leq-\frac{\alpha(x)(\Gamma(x)-x)}{\Gamma(x)^{2}}
$$

since $S$ is increasing. Differentiating (5.38) with respect to $x$, we also compute

$$
\frac{\partial}{\partial x} \mathcal{L} g(x, z)=\frac{\alpha^{\prime}(x)(z-x)-\alpha(x)}{z^{2}}+m^{\prime}(x) \int_{z}^{\infty} \frac{u-2 x}{u^{3} S(u)} d u-\left[m(x)+S^{\prime}(x)\right] \int_{z}^{\infty} \frac{2 d u}{u^{3} S(u)},
$$

for $z \geq x$. Denoting $A:=\left(\alpha m^{\prime}-\alpha^{\prime} m\right)(\Gamma-I d)+\alpha m$, the two previous estimates lead to

$$
\frac{\partial}{\partial x} \mathcal{L} g(x, \Gamma(x)) \leq-\frac{A(x)}{m(x) \Gamma(x)^{2}}-\left[m(x)+S^{\prime}(x)\right] \int_{\Gamma(x)}^{\infty} \frac{2 d u}{u^{3} S(u)} .
$$

Introducing $B:=\alpha m^{\prime}-\alpha^{\prime} m$ and observing that $x \leq \Gamma(x) \leq 2 x$, we obtain

$$
A(x) \geq \alpha(x) m(x) \mathbf{1}_{\{B(x) \geq 0\}}+(x B(x)+\alpha(x) m(x)) \mathbf{1}_{\{B(x)<0\}} .
$$

Introducing finally $C: x \mapsto x B(x)+\alpha(x) m(x)$, we compute $C(0)=2 \alpha(0) \geq 0$ and

$$
C^{\prime}(x)=2 \alpha(x) m^{\prime}(x)+x\left(\alpha(x) m^{\prime \prime}(x)-\alpha^{\prime \prime}(x) m(x)\right) \geq 0,
$$

because $m^{\prime \prime} \geq 0$ and $\alpha^{\prime \prime} \leq 0$. Therefore $C$ is non-negative, and, according to (5.58), A is also non-negative. As a consequence, combining $m>0$ and (5.57), we deduce that 
$\frac{\partial}{\partial x} \mathcal{L} g(x, \Gamma(x))<0$. Using Lemma 5.1, we have $\frac{\partial}{\partial z} \mathcal{L} g(x, \Gamma(x))>0$, and (5.56) implies that $\Gamma^{\prime}(x)>0$.

Therefore $\Gamma$ is increasing on the set $\{x>0, \Gamma(x)>x\}$. But it is also increasing on the interior of the set $\{x>0, \Gamma(x)=x\}$. Since $\Gamma$ is continuous, it is increasing on $(0,+\infty)$.

(ii) We have $\Gamma^{\infty}:=\sup \{x \geq 0 ; \Gamma(x)>x\}<\infty$.

The arguments used here are very close to the ones in the proof of Proposition 4.3 in [5]. However, our conclusions cannot be deduced from theirs since the involved computations are different and we need to detail this proof.

From the definition of the scale function (2.9), we compute:

$$
S(x)=S(1)+\frac{S^{\prime}(x)}{\alpha(x)}-\frac{S^{\prime}(1)}{\alpha(1)}-\int_{1}^{x}\left(\frac{1}{\alpha}\right)^{\prime}(u) S^{\prime}(u) d u, \quad x>0 .
$$

We then distinguish two cases depending on the explosion of the last term in the previous expression.

Case 1: $\int_{1}^{\infty}(1 / \alpha)^{\prime}(u) S^{\prime}(u) d u>-\infty$.

Then $S(x)=\frac{S^{\prime}(x)}{\alpha(x)}+O(1)$ for $x$ large enough. Recalling that $\mathcal{L} S=0$, we compute

$$
\begin{aligned}
\int_{x}^{\infty} \frac{d u}{u^{2} S(u)} & =\int_{x}^{\infty} \frac{d u}{u^{2}\left(\frac{S^{\prime}(u)}{\alpha(u)}+O(1)\right)}=\int_{x}^{\infty} \frac{\alpha(u)}{u^{2} S^{\prime}(u)} \frac{d u}{1+O\left(\frac{\alpha(u)}{S^{\prime}(u)}\right)} \\
& =\int_{x}^{\infty} \frac{\alpha(u) d u}{u^{2} S^{\prime}(u)}+O\left(\int_{x}^{\infty} \frac{\alpha^{2}(u)}{u^{2}\left[S^{\prime}(u)\right]^{2}}\right)
\end{aligned}
$$

for $x$ large enough. Integrating by parts, we observe that

$$
\int_{x}^{\infty} \frac{\alpha(u) d u}{u^{2} S^{\prime}(u)}=\frac{1}{x^{2} S^{\prime}(x)}-2 \int_{x}^{\infty} \frac{d u}{u^{3} S^{\prime}(u)}, \quad x>1
$$

We now prove that $\frac{x \alpha^{2}(x)}{S^{\prime}(x)} \rightarrow 0$ when $x \rightarrow \infty$.

Indeed, since $\alpha(1)>0$ by (2.12), and since $\alpha$ is non-decreasing, we get $S^{\prime}(x) \geq e^{(x-1) \alpha(1)}$, for any $x \geq 1$. On the other hand, since $\alpha$ is concave, we also have $0 \leq \alpha(x) \leq x \alpha^{\prime}(0)$, so that:

$$
0 \leq \frac{x \alpha^{2}(x)}{S^{\prime}(x)} \leq \frac{x^{3}\left[\alpha^{\prime}(0)\right]^{2}}{e^{(x-1) \alpha(1)}} \rightarrow 0 \quad \text { when } \quad x \rightarrow \infty .
$$

As a consequence, we get

$$
\int_{x}^{\infty} \frac{d u}{u^{2} S(u)}=\frac{1}{x^{2} S^{\prime}(x)}-2 \int_{x}^{\infty} \frac{d u}{u^{3} S^{\prime}(u)}+\circ\left(\int_{x}^{\infty} \frac{d u}{u^{3} S^{\prime}(u)}\right) .
$$

Integrating by parts again, we finally compute

$$
\int_{x}^{\infty} \frac{d u}{u^{2} S(u)}=\frac{1}{x^{2} S^{\prime}(x)}-\frac{2}{\alpha(x) x^{3} S^{\prime}(x)}+\circ\left(\frac{1}{\alpha(x) x^{3} S^{\prime}(x)}\right)
$$


and similarly we get

$$
\int_{x}^{\infty} \frac{d u}{u^{3} S(u)}=\frac{1}{x^{3} S^{\prime}(x)}-\frac{3}{\alpha(x) x^{4} S^{\prime}(x)}+\circ\left(\frac{1}{\alpha(x) x^{4} S^{\prime}(x)}\right),
$$

Plugging these estimates in the expression of $\mathcal{L} g$ given by (5.34) leads to:

$$
\begin{aligned}
\mathcal{L} g(x, x) & =\frac{1}{x^{2}}+\left[2 S^{\prime}(x)-\alpha(x) S(x)\right] \int_{x}^{\infty} \frac{d u}{u^{2} S(u)}-\left[S(x)+2 x S^{\prime}(x)-x \alpha(x) S(x)\right] \int_{x}^{\infty} \frac{2 d u}{u^{3} S(u)} \\
& =\frac{1}{x^{2}}+\left(S^{\prime}(x)+O(\alpha(x))\right)\left(\frac{1}{x^{2} S^{\prime}(x)}-\frac{2}{\alpha(x) x^{3} S^{\prime}(x)}+\circ\left(\frac{1}{\alpha(x) x^{3} S^{\prime}(x)}\right)\right) \\
& -2\left(x S^{\prime}(x)+\frac{S^{\prime}(x)}{\alpha(x)}+O(x \alpha(x))\right)\left(\frac{1}{x^{3} S^{\prime}(x)}-\frac{3}{\alpha(x) x^{4} S^{\prime}(x)}+\circ\left(\frac{1}{\alpha(x) x^{4} S^{\prime}(x)}\right)\right) .
\end{aligned}
$$

Using the fact that $\frac{x \alpha^{2}(x)}{S^{\prime}(x)} \rightarrow 0$ as $x \rightarrow \infty$, we get:

$$
\mathcal{L} g(x, x)=\frac{2}{\alpha(x) x^{3}}+\circ\left(\frac{1}{\alpha(x) x^{3}}\right) .
$$

Hence $\mathcal{L} g(x, x)>0$ and therefore $\Gamma(x)=x$ for $x$ large enough, so that $\Gamma_{\infty}<\infty$.

Case 2: $\int_{1}^{\infty}(1 / \alpha)^{\prime}(u) S^{\prime}(u) d u=-\infty$.

For $x$ large enough, we have

$$
S(x)=\frac{S^{\prime}(x)}{\alpha(x)}\left[1-\left(\frac{1}{\alpha}\right)^{\prime}(x)+\circ\left(\left(\frac{1}{\alpha}\right)^{\prime}(x)\right)\right],
$$

so that

$$
\begin{aligned}
\int_{x}^{\infty} \frac{d u}{u^{2} S(u)} & =\int_{x}^{\infty} \frac{\alpha(u)}{u^{2} S^{\prime}(u)}\left[1+\left(\frac{1}{\alpha}\right)^{\prime}(u)+\circ\left(\left(\frac{1}{\alpha}\right)^{\prime}(u)\right)\right] d u \\
& =\frac{1}{x^{2} S^{\prime}(x)}-\int_{x}^{\infty} \frac{2 d u}{u^{3} S^{\prime}(u)}-\int_{x}^{\infty} \frac{\alpha^{\prime}(u) d u}{u^{2} \alpha(u) S^{\prime}(u)}+\circ\left(\int_{x}^{\infty} \frac{\alpha(u)+u \alpha^{\prime}(u)}{u^{3} \alpha(u) S^{\prime}(u)} d u\right) .
\end{aligned}
$$

Noticing that $0 \leq x \alpha^{\prime}(x) \leq \alpha(x)$ for $x>0$, since $\alpha$ is concave, we have:

$$
\circ\left(\int_{x}^{\infty} \frac{\alpha(u)+u \alpha^{\prime}(u)}{u^{3} \alpha(u) S^{\prime}(u)} d u\right)=\circ\left(\int_{x}^{\infty} \frac{d u}{u^{3} S^{\prime}(u)}\right) .
$$

Integrating by parts, we finally get

$$
\int_{x}^{\infty} \frac{d u}{u^{2} S(u)}=\frac{1}{x^{2} S^{\prime}(x)}-\frac{2}{x^{3} \alpha(x) S^{\prime}(x)}-\frac{\alpha^{\prime}(x)}{x^{2} \alpha^{2}(x) S^{\prime}(x)}+\circ\left(\frac{1}{x^{3} \alpha(x) S^{\prime}(x)}\right)
$$

where the third term in the previous expansion might be negligible or not (depending on $\alpha$ ). Similarly, we compute:

$$
\int_{x}^{\infty} \frac{d u}{u^{3} S(u)}=\frac{1}{x^{3} S^{\prime}(x)}-\frac{3}{x^{4} \alpha(x) S^{\prime}(x)}-\frac{\alpha^{\prime}(x)}{x^{3} \alpha^{2}(x) S^{\prime}(x)}+\circ\left(\frac{1}{x^{4} \alpha(x) S^{\prime}(x)}\right),
$$


so that:

$$
\begin{aligned}
\mathcal{L} g(x, x) & =\frac{1}{x^{2}}+\left[2 S^{\prime}(x)-\alpha(x) S(x)\right] \int_{x}^{\infty} \frac{d u}{u^{2} S(u)}-\left[S(x)+2 x S^{\prime}(x)-x \alpha(x) S(x)\right] \int_{x}^{\infty} \frac{2 d u}{u^{3} S(u)} \\
& =\frac{1}{x^{2}}-2\left(x S^{\prime}(x)-x \frac{\alpha^{\prime}(x)}{\alpha^{2}(x)} S^{\prime}(x)+\frac{S^{\prime}(x)}{\alpha(x)}\right)\left(\frac{1}{x^{3} S^{\prime}(x)}-\frac{3}{x^{4} \alpha(x) S^{\prime}(x)}-\frac{\alpha^{\prime}(x)}{x^{3} \alpha^{2}(x) S^{\prime}(x)}\right) \\
& +\left(S^{\prime}(x)-\frac{\alpha^{\prime}(x)}{\alpha^{2}(x)} S^{\prime}(x)\right)\left(\frac{1}{x^{2} S^{\prime}(x)}-\frac{2}{x^{3} \alpha(x) S^{\prime}(x)}-\frac{\alpha^{\prime}(x)}{x^{2} \alpha^{2}(x) S^{\prime}(x)}\right)+\circ\left(\frac{1}{x^{3} \alpha(x)}\right) \\
& =\frac{2}{x^{3} \alpha(x)}+\frac{2 \alpha^{\prime}(x)}{x^{2} \alpha^{2}(x)}+\circ\left(\frac{1}{x^{3} \alpha(x)}\right),
\end{aligned}
$$

where the second term might be or not negligible. In any case, we see that for sufficiently large $x, \mathcal{L} g(x, x)>0$, so that $\Gamma(x)=x$. Therefore, $\Gamma_{\infty}<\infty$ also holds in this case.

\subsection{Proof of Proposition 5.3}

This section is dedicated to the proof of Proposition 5.3. As already explained, this proof uses the same ideas as the one developed in [5]. However, because of the specificity of (5.47), the properties of the flow are different and the analysis needs to be adapted to our framework. We will try to follow their notations and point out in the proofs the parts that are identical to their paper, but we choose to rewrite them for the sake of completeness.

First, for the convenience of the reader, we recall ODE (5.47) that $\gamma$ needs to satisfy:

$$
\gamma^{\prime}(x)=\frac{\gamma(x)^{2} \mathcal{L} g(x, \gamma(x))}{\left(\frac{2 x}{\gamma(x)}-1\right)\left(1-\frac{S(x)}{S \circ \gamma(x)}\right)}, \quad x>0 .
$$

Let us first define

$$
\mathbf{D}^{-}:=\{x>0: \mathcal{L} g(x, x)<0\},
$$

and, for all $x_{0} \in \mathbf{D}^{-}$, we introduce

$$
d\left(x_{0}\right):=\sup \left\{x \leq x_{0}: \mathcal{L} g(x, x) \geq 0\right\} \quad \text { and } \quad u\left(x_{0}\right):=\inf \left\{x \geq x_{0} ; \mathcal{L} g(x, x) \geq 0\right\}
$$

with the convention that $d\left(x_{0}\right)=0$ if $\left\{x \leq x_{0}: \mathcal{L} g(x, x) \geq 0\right\}=\emptyset$. Observe that Proposition 5.2 ensures that $u\left(x_{0}\right) \leq \Gamma^{\infty}<\infty$. Since $\mathcal{L} g$ is continuous and $x_{0} \in \mathbf{D}^{-}$we must have $d\left(x_{0}\right)<x_{0}<u\left(x_{0}\right)<\infty$.

For any $x_{0} \in \mathbf{D}^{-}$and $z_{0}>x_{0}$, we denote by $\gamma_{x_{0}}^{z_{0}}$ the maximal solution of the Cauchy problem (5.59) complemented by the additional condition $\gamma\left(x_{0}\right)=z_{0}$, and we denote by

$I_{x_{0}}^{z_{0}}:=\left(\ell_{x_{0}}^{z_{0}}, r_{x_{0}}^{z_{0}}\right)$ the corresponding (open) interval of definition of $\gamma_{x_{0}}^{z_{0}}$. Since the right-hand side of ODE (5.59) is locally Lipschitz on either one of the sets $\{(x, \gamma), 0<2 x<\gamma\}$ or $\{(x, \gamma), x<\gamma<2 x\}$ but is not defined on the set $\{(x, \gamma), 2 x=\gamma\}$, the maximal solution will be defined as long as $(x, \gamma(x))$ remains in one of those two sets. Since $\Gamma\left(x_{0}\right)<2 x_{0}$, we restrict our attention to conditions $\gamma\left(x_{0}\right)=z_{0}$ satisfying $x_{0}<z_{0}<2 x_{0}$.

The next lemma provides useful additional properties of the maximal solutions described above and their respective domains of definitions. 
Lemma 5.2 Assume that $\alpha$ satisfies Conditions (2.12) and let $x_{0} \in \mathbf{D}^{-}$be fixed.

(i) For all $z_{0} \in\left(x_{0}, 2 x_{0}\right)$, $\ell_{x_{0}}^{z_{0}} \leq d\left(x_{0}\right)$, we have $\lim _{x \rightarrow \ell_{x_{0}}^{z_{0}}} \gamma_{x_{0}}^{z_{0}}(x)=\ell_{x_{0}}^{z_{0}}$ and, if $\ell_{x_{0}}^{z_{0}}>0$, we get $\mathcal{L} g\left(\ell_{x_{0}}^{z_{0}}, \ell_{x_{0}}^{z_{0}}\right) \geq 0$

(ii) for all $z_{0} \in\left(x_{0}, \Gamma\left(x_{0}\right)\right], \mathcal{L} g\left(x, \gamma_{x_{0}}^{z_{0}}(x)\right)<0$ for any $x \in\left(x_{0}, r_{x_{0}}^{z}\right)$;

(iii) there exists $a_{0} \in\left(x_{0}, 2 x_{0}\right)$ such that for any $z_{0} \in\left[a_{0}, 2 x_{0}\right), \mathcal{L} g\left(x, \gamma_{x_{0}}^{z_{0}}(x)\right)>0$ for any $x \in\left(x_{0}, r_{x_{0}}^{z_{0}}\right)$.

Proof. We fix $x_{0} \in \mathbf{D}^{-}$and prove each property separately.

(i) Let us fix $z_{0} \in\left(x_{0}, 2 x_{0}\right)$. The right-hand side of (5.59) is locally Lipschitz as long as $0<x<\gamma_{x_{0}}^{z_{0}}(x)<2 x$, so that this last estimate holds for any $x \in I_{x_{0}}^{z_{0}}$. We intend to prove that $\gamma_{x_{0}}^{z}$ hits the diagonal $\{(x, z) ; x=z\}$ at the left hand side $\ell_{x_{0}}^{z_{0}}$ of $I_{x_{0}}^{z_{0}}$.

For this purpose, let us first prove that, for any $\zeta \in\left(0, x_{0}\right)$, the graph of $\gamma_{x_{0}}^{z_{0}}$ restricted to $\left[\zeta, x_{0}\right]$ cannot come too close to $\{(x, z) ; 2 x=z\}$. Since $\Gamma(x)<2 x$ for $x>0$ and $\Gamma$ and $\mathcal{L} g$ are continuous, there exist $\varepsilon>0$ and $\delta>0 \in(0, \zeta)$ such that $\mathcal{L} g \geq \varepsilon$ on the compact set $\left\{(x, z) ; x \in\left[\zeta, x_{0}\right]\right.$ and $\left.z \in[2 x-\delta, 2 x]\right\}$. Observe that, for $x \in\left[\zeta, x_{0}\right]$ such that $\gamma_{x_{0}}^{z_{0}}(x) \in\left[\max \left(2 x-\delta, \frac{4 x}{2+\zeta^{2} \varepsilon}\right), 2 x\right)$, we get from (5.59) that

$$
\left(\gamma_{x_{0}}^{z_{0}}\right)^{\prime}(x) \geq \frac{\gamma_{x_{0}}^{z_{0}}(x)^{2} \mathcal{L} g\left(x, \gamma_{x_{0}}^{z_{0}}(x)\right)}{\frac{2 x}{\gamma_{x_{0}}^{z_{0}}(x)}-1} \geq \frac{2 \gamma_{x_{0}}^{z_{0}}(x)^{2} \varepsilon}{\zeta^{2} \varepsilon} \geq 2,
$$

where, for the last inequality, we used $\gamma(x) \geq x \geq \zeta$. Hence, the function $x \mapsto 2 x-\gamma_{x_{0}}^{z_{0}}(x)$ is non-increasing on the set $\left\{x \in\left[\zeta, x_{0}\right] \cap I_{x_{0}}^{z_{0}} ; \gamma_{x_{0}}^{z_{0}}(x) \in\left[\max \left(2 x-\delta, \frac{4 x}{2+\zeta^{2} \varepsilon}\right), 2 x\right)\right\}$. Therefore, by arbitrariness of $\zeta>0$, the graph of $\gamma_{x_{0}}^{z_{0}}$ restricted to $\left(\ell_{x_{0}}^{z_{0}}, x_{0}\right]$ stays away from $\{(x, z) ; 2 x=z\}$ and $\gamma_{x_{0}}^{z_{0}}$ necessarily hits the diagonal $\{(x, z) ; x=z\}$ at the left hand side $\ell_{x_{0}}^{z_{0}}$ of the maximal interval $I_{x_{0}}^{z_{0}}$.

On the other hand, we observe from (5.59) that $\gamma_{x_{0}}^{z_{0}}$ is non-increasing at the points $x$ satisfying $\left(x, \gamma_{x_{0}}^{z_{0}}(x)\right) \in \Gamma^{-}$and therefore $\ell_{x_{0}}^{z_{0}} \notin \mathbf{D}^{-}$by minimality of $I_{x_{0}}^{z_{0}}$. Since $\left(d\left(x_{0}\right), u\left(x_{0}\right)\right) \subset$ $\mathbf{D}^{-}$, we get $\ell_{x_{0}}^{z_{0}} \leq d\left(x_{0}\right)$ and $\mathcal{L} g\left(\ell_{x_{0}}^{z_{0}}, \ell_{x_{0}}^{z_{0}}\right) \geq 0$, or equivalently $\Gamma\left(\ell_{x_{0}}^{z_{0}}\right)=\ell_{x_{0}}^{z_{0}}$.

It still remains to prove properly that $\lim _{x \rightarrow \ell_{x_{0}}} \gamma_{x_{0}}^{z_{0}}(x)=\ell_{x_{0}}^{z_{0}}$. Assume first that $\ell_{x_{0}}^{z_{0}}>0$. Notice from (5.59) that $\gamma_{x_{0}}^{z_{0}}$ is non-decreasing if $\left(x, \gamma_{x_{0}}^{z_{0}}(x)\right) \in \Gamma^{+}$, and $x \leq \gamma_{x_{0}}^{z_{0}}(x) \leq \Gamma(x)$ otherwise. Since $\Gamma$ is also non-decreasing, $\tilde{\gamma}_{x_{0}}^{z_{0}}:=\max \left(\gamma_{x_{0}}^{z_{0}}, \Gamma\right)$ is a non-decreasing function defined on $\left(\ell_{x_{0}}^{z_{0}}, x_{0}\right]$, which therefore admits a limit at $\ell_{x_{0}}^{z_{0}}$. Since $\ell_{x_{0}}^{z_{0}}>0$, we have $\lim _{x \rightarrow \ell_{x_{0}}^{z_{0}}} \tilde{\gamma}_{x_{0}}^{z_{0}}(x)<2 \ell_{x_{0}}^{z_{0}}$ as observed above, so that, combining the maximality of $I_{x_{0}}^{z_{0}}$ with $\Gamma\left(\ell_{x_{0}}^{z_{0}}\right)=\ell_{x_{0}}^{z_{0}}$, we obtain $\lim _{x \rightarrow \ell_{x_{0}}^{z_{0}}} \tilde{\gamma}_{x_{0}}^{z_{0}}(x)=\ell_{x_{0}}^{z_{0}}$. Since $x \leq \gamma_{x_{0}}^{z_{0}}(x) \leq \tilde{\gamma}_{x_{0}}^{z_{0}}(x)$ for $x \in\left(\ell_{x_{0}}^{z_{0}}, x_{0}\right]$, we have $\lim _{x \rightarrow \ell_{x_{0}}^{z_{0}}} \gamma_{x_{0}}^{z_{0}}(x)=\ell_{x_{0}}^{z_{0}}$. Finally, if $\ell_{x_{0}}^{z_{0}}=0$, since $x<\gamma_{x_{0}}^{z_{0}}(x)<2 x$, we also have the result.

(ii) Let us fix $z_{0} \in\left(x_{0}, \Gamma\left(x_{0}\right)\right)$. As already observed, the dynamics of (5.59) imply that $\gamma_{x_{0}}^{z_{0}}$ is non-increasing in the neighborhood of any point $x$ such that $\left(x, \gamma_{x_{0}}^{z_{0}}(x)\right) \in \operatorname{Int}\left(\Gamma^{-}\right)$. On the other hand, Proposition 5.2 tells us that the function $\Gamma$ is increasing on $\left[x_{0},+\infty\right)$. Hence $x \mapsto\left(x, \gamma_{x_{0}}^{z_{0}}(x)\right)$ remains in $\operatorname{Int}\left(\Gamma^{-}\right)$on $\left[x_{0}, r_{x_{0}}^{z_{0}}\right)$. 
We consider now the case where $z_{0}=\Gamma\left(x_{0}\right)$. Since $\Gamma\left(x_{0}\right)>x_{0}$, the proof of Proposition 5.2 (i) tells us that $\Gamma^{\prime}$ is positive on a neighborhood of $x_{0}$. Since $z_{0}=\Gamma\left(x_{0}\right)$, we deduce from (5.59) that $\left(\gamma_{x_{0}}^{z_{0}}\right)^{\prime}\left(x_{0}\right)=0$, and the exact same reasoning as above implies that $x \mapsto\left(x, \gamma_{x_{0}}^{z_{0}}(x)\right) \in \operatorname{Int}\left(\Gamma^{-}\right)$on $\left(x_{0}, r_{x_{0}}^{z_{0}}\right)$.

(iii) Recall that $\Gamma^{\infty}<\infty$. Therefore, as in (i), there exist $\varepsilon>0$ and $\delta \in(0,1)$ such that $\mathcal{L} g \geq \varepsilon$ on $\left\{(x, z) ; x \in\left[x_{0}, \Gamma^{\infty}\right]\right.$ and $\left.z \in[(2-\delta) x, 2 x]\right\}$. Let $b:=\min \left(x_{0}^{2} \varepsilon, \delta\right)$. From (5.59), we see that if $x \in\left[x_{0}, \Gamma^{\infty}\right]$ and $\gamma_{x_{0}}^{z}(x) \in[(2-b) x, 2 x)$, then $\left(\gamma_{x_{0}}^{z}\right)^{\prime}(x) \geq \frac{2-b}{b} x_{0}^{2} \varepsilon \geq 2-b$. We denote $a_{0}:=(2-b) x_{0}$ and fix $z \in\left[a_{0}, 2 x_{0}\right)$. We deduce from the previous reasoning that we must have

$$
\gamma_{x_{0}}^{z}(x) \geq z+\int_{x_{0}}^{x}\left(\gamma_{x_{0}}^{z}\right)^{\prime}(u) d u \geq z+(2-b)\left(x-x_{0}\right)>(2-b) x>\Gamma(x),
$$

for $x \in\left[x_{0}, \min \left(r_{x_{0}}^{z}, \Gamma^{\infty}\right)\right)$. If ever $r_{x_{0}}^{z} \leq \Gamma^{\infty}$, we just obtained the announced result and, if ever $r_{x_{0}}^{z}>\Gamma^{\infty}$, we complete the proof noticing that the maximality of $I_{x_{0}}^{z}$ implies that $\gamma_{x_{0}}^{z}(x)>x=\Gamma(x)$ for $x \geq \Gamma^{\infty}$.

We now construct the stopping boundary $\gamma$ by selecting one of the previous maximal solutions. For a given $x_{0} \in \mathbf{D}^{-}$, let

$$
\begin{aligned}
& \mathbf{Z}\left(x_{0}\right):=\left\{z \in\left(x_{0}, 2 x_{0}\right) ; \mathcal{L} g\left(x, \gamma_{x_{0}}^{z}(x)\right)<0 \text { for some } x \in\left[x_{0}, r_{x_{0}}^{z}\right)\right\} \\
& z^{*}\left(x_{0}\right):=\sup \mathbf{Z}\left(x_{0}\right) .
\end{aligned}
$$

Moreover, whenever $z^{*}\left(x_{0}\right)<2 x_{0}$, we denote

$$
\gamma_{x_{0}}^{*}:=\gamma_{x_{0}}^{z^{*}\left(x_{0}\right)}, \ell_{x_{0}}^{*}:=\ell_{x_{0}}^{z^{*}\left(x_{0}\right)}, r_{x_{0}}^{*}:=r_{x_{0}}^{z^{*}\left(x_{0}\right)}, \text { and } I_{x_{0}}^{*}:=\left(\ell_{x_{0}}^{*}, r_{x_{0}}^{*}\right)
$$

The next lemma provides useful properties on the function $\gamma^{*}$ and its domain of definition. In particular, it discusses its dependance with respect to the starting point $x_{0}$.

Lemma 5.3 Assume that $\alpha$ satisfies Conditions (2.12) and let $x_{0}$ be arbitrary in $\mathbf{D}^{-}$. Then, the following holds.

(i) $z^{*}\left(x_{0}\right) \in\left(\Gamma\left(x_{0}\right), 2 x_{0}\right)$ and $\gamma_{x_{0}}^{*}$ has a positive derivative on the interval $I_{x_{0}}^{*}$.

(ii) $\left(d\left(x_{0}\right), u\left(x_{0}\right)\right) \subset I_{x_{0}}^{*}$ and $\lim _{x \rightarrow r_{x_{0}}^{*}} \gamma_{x_{0}}^{*}(x)=r_{x_{0}}^{*} \leq \Gamma^{\infty}$ with equality if $u\left(x_{0}\right)=\Gamma^{\infty}$.

(iii) For $x_{0}, x_{1} \in \mathbf{D}^{-}$, we have either $I_{x_{0}}^{*} \cap I_{x_{1}}^{*}=\emptyset$, or $I_{x_{0}}^{*}=I_{x_{1}}^{*}$ and $\gamma_{x_{0}}^{*}=\gamma_{x_{1}}^{*}$.

Proof. We fix $x_{0} \in \mathbf{D}^{-}$and prove each assertion separately. The proofs of points (i) and (iii) are very close to the proof of Lemma 5.2 in [5], but we rewrite and adapt them here.

(i) Lemma 5.2 (iii) ensures the existence of $a_{0}<2 x_{0}$ such that $\mathcal{L} g\left(x, \gamma_{x_{0}}^{z}(x)\right)>0$ for any $x \geq x_{0}$ and $z \geq a_{0}$. By definition of $z^{*}\left(x_{0}\right)$, we deduce that $z^{*}\left(x_{0}\right) \leq a_{0}<2 x_{0}$. 
Since $x_{0} \in \mathbf{D}^{-}$, we obtain from Lemma 5.2 (ii) that $\Gamma\left(x_{0}\right) \in \mathbf{Z}\left(x_{0}\right)$ and deduce that $\Gamma\left(x_{0}\right) \leq z^{*}\left(x_{0}\right)$.

In order to prove that $z^{*}\left(x_{0}\right) \in\left(\Gamma\left(x_{0}\right), 2 x_{0}\right)$, we now assume that $z^{*}\left(x_{0}\right)=\Gamma\left(x_{0}\right)$ and work towards a contradiction. Since $\mathcal{L} g$ is continuous, $\mathbf{D}^{-}$is an open set and there exists $\varepsilon>0$ such that $\left(x_{0}, x_{0}+2 \varepsilon\right) \subset \mathbf{D}^{-} \cap\left(x_{0}, r_{x_{0}}^{*}\right)$ and $d(x)=d\left(x_{0}\right)$ for any $x \in\left(x_{0}, x_{0}+\varepsilon\right)$. Let us denote $x_{\varepsilon}:=x_{0}+\varepsilon \in \mathbf{D}^{-}$and $z_{\varepsilon}:=\Gamma\left(x_{\varepsilon}\right)>\Gamma\left(x_{0}\right)$. By Lemma 5.2 (i), we have $\ell_{x_{\varepsilon}}^{z_{\varepsilon}^{\varepsilon}} \leq d\left(x_{0}\right)<x_{0}$, and it follows from Lemma 5.2 (ii) and the dynamics of (5.59) that $\gamma_{x_{\varepsilon}}^{z_{\varepsilon}}$ is decreasing on $\left(x_{0}, r_{x_{\varepsilon}}^{z_{\varepsilon}^{\varepsilon}}\right)$. Therefore, we compute

$$
\gamma_{x_{\varepsilon}}^{z_{\varepsilon}}\left(x_{0}\right)>\gamma_{x_{\varepsilon}}^{z_{\varepsilon}}\left(x_{\varepsilon}\right)=\Gamma\left(x_{\varepsilon}\right)>\Gamma\left(x_{0}\right)=z^{*}
$$

On the other hand, since $\gamma_{x_{0}}^{\gamma_{x_{\varepsilon}}^{\varepsilon_{\varepsilon}}\left(x_{0}\right)}\left(x_{\varepsilon}\right)=z^{\varepsilon}=\Gamma\left(x_{\varepsilon}\right)$, Lemma 5.2 (ii) ensures that $\gamma_{x_{\varepsilon}}^{z^{\varepsilon}}\left(x_{0}\right) \in$ $\mathbf{Z}\left(x_{0}\right)$, leading to $z^{*} \geq \gamma_{x_{\varepsilon}}^{z^{\varepsilon}}\left(x_{0}\right) \in \mathbf{Z}\left(x_{0}\right)$, which contradicts (5.65).

The same line of argument implies also that $\left(x, \gamma^{*}(x)\right) \in \operatorname{Int}\left(\Gamma^{+}\right)$for any $x \in I_{x_{0}}^{*}$. We deduce from the dynamics of (5.59) that $\gamma^{*}$ has a positive derivative on $I_{x_{0}}^{*}$, and in particular $\lim _{x \rightarrow r_{x_{0}}^{*}} \gamma^{*}(x)$ exists.

(ii) For any $z \in \mathbf{Z}\left(x_{0}\right)$, since $\gamma_{x_{0}}^{z}$ is non-increasing in $\Gamma^{-}$, we deduce that $\lim _{x \rightarrow r_{x_{0}}} \gamma_{x_{0}}^{z}(x)=$ $r_{x_{0}}^{z} \leq \Gamma^{\infty}$. Let us write $r_{0}:=\sup \left\{r_{x_{0}}^{z} ; z \in \mathbf{Z}\left(x_{0}\right)\right\} \leq \Gamma^{\infty}$. Let us first prove that $r_{0} \geq u\left(x_{0}\right)$. Assume on the contrary that $r_{0}<u\left(x_{0}\right)$, so that $\Gamma\left(r_{0}\right)>r_{0}$. Let fix $z \in\left(r_{0}, \Gamma\left(r_{0}\right)\right)$. By Lemma $5.2(\mathrm{i}), \ell_{r_{0}}^{z} \leq d\left(x_{0}\right)$, so that $x_{0} \in I_{r_{0}}^{z}$ and, since Lemma 5.2 (ii) implies that $\mathcal{L} g\left(x, \gamma_{r_{0}}^{z}(x)\right)<0$ for $x>r_{0}$, we deduce that $\gamma_{r_{0}}^{z}\left(x_{0}\right) \in \mathbf{Z}\left(x_{0}\right)$. This contradicts the definition of $r_{0}$ since $z \in\left(r_{0}, \Gamma\left(r_{0}\right)\right)$ implies that $r_{x_{0}}^{\gamma_{r_{0}}^{z}\left(x_{0}\right)}=r_{r_{0}}^{z}>r_{0}$. In conclusion, $r_{0} \geq u\left(x_{0}\right)$. Besides, Lemma 5.2 (i) implies that $\ell_{x_{0}}^{*} \leq d\left(x_{0}\right)$, and we intend to prove that $r_{0}=r_{x_{0}}^{*}$ in order to derive $\left(d\left(x_{0}\right), u\left(x_{0}\right)\right) \subset I_{x_{0}}^{*}$.

First, we derive the existence of a sequence $\left(z_{n}\right) \in \mathbf{Z}\left(x_{0}\right)$ such that $z_{n} \rightarrow z^{*}\left(x_{0}\right)$ and $r_{x_{0}}^{z_{n}} \rightarrow$ $r_{0}$. Combining the one-to-one property of the flow with the property that $\lim _{x \rightarrow r_{x_{0}}^{z}} \gamma_{x_{0}}^{z}(x)=$ $r_{x_{0}}^{z}$ for $z \in \mathbf{Z}\left(x_{0}\right)$, we deduce that $z \mapsto r_{x_{0}}^{z}$ is non-decreasing on $\mathbf{Z}\left(x_{0}\right)$. Hence, if $z^{*}\left(x_{0}\right) \notin$ $\mathbf{Z}\left(x_{0}\right)$, any sequence $\left(z_{n}\right)$ valued in $\mathbf{Z}\left(x_{0}\right)$ such that $z_{n} \rightarrow z^{*}\left(x_{0}\right)$ satisfies also $\sup \left\{r_{x_{0}}^{z_{n}} ; n \in\right.$ $\mathbb{N}\}=\sup \left\{r_{x_{0}}^{z} ; z \in \mathbf{Z}\left(x_{0}\right)\right\}=r_{0}$ and thus the required property. If ever $z^{*}\left(x_{0}\right) \in \mathbf{Z}\left(x_{0}\right)$, we simply pick the sequence $z_{n}:=z^{*}\left(x_{0}\right)$, for any $n \in \mathbb{N}$.

We now prove that $r_{0}=r_{x_{0}}^{*}$. Let $z \in\left(r_{0}, 2 r_{0}\right)$ be arbitrary. Up to a subsequence, we have by construction $I_{x_{0}}^{z_{n}} \cap I_{r_{0}}^{z} \neq \emptyset$ for any $n \in \mathbb{N}$. We know that $\lim _{x \rightarrow r_{x_{0}}^{z_{n}}} \gamma_{x_{0}}^{z_{n}}(x)=r_{x_{0}}^{z_{n}}$ and $\gamma_{r_{0}}^{z}\left(r_{x_{0}}^{z_{n}}\right)>r_{x_{0}}^{z_{n}}$ since $r_{r_{0}}^{z}>r_{0} \geq r_{x_{0}}^{z_{n}}$, for any $n \in \mathbb{N}$. Hence, the one-to-one property of the flow ensures that $\gamma_{r_{0}}^{z}(x)>\gamma_{x_{0}}^{z_{n}}(x)$ for any $x \in I_{x_{0}}^{z_{n}} \cap I_{r_{0}}^{z}$ and $n \in \mathbb{N}$. By Lemma 5.2 (i), $\lim _{x \rightarrow \ell_{r_{0}}^{z}} \gamma_{r_{0}}^{z}(x)=\ell_{r_{0}}^{z}$, so that $x_{0} \in I_{x_{0}}^{z_{n}} \cap I_{r_{0}}^{z}$. Since $\left(z_{n}\right) \in \mathbf{Z}\left(x_{0}\right)$ converges to $z^{*}\left(x_{0}\right)=\sup \mathbf{Z}\left(x_{0}\right)$, we deduce that $\gamma_{r_{0}}^{z}\left(x_{0}\right) \geq \gamma_{x_{0}}^{*}\left(x_{0}\right)=z^{*}\left(x_{0}\right) \geq z^{n}=\gamma_{x_{0}}^{z_{n}}\left(x_{0}\right)$, for any 
$n \in \mathbb{N}$. Hence, the one-to-one property of the flow implies that

$$
2 x>\gamma_{r_{0}}^{z}(x) \geq \gamma_{x_{0}}^{*}(x) \geq \gamma_{x_{0}}^{z_{n}}(x), \quad x \in\left[x_{0}, r_{x_{0}}^{z_{n}} \wedge r_{x_{0}}^{*}\right), \quad n \in \mathbb{N} .
$$

Therefore $r_{x_{0}}^{*} \geq r_{x_{0}}^{z_{n}}$ for $n \in \mathbb{N}$, and, passing to the limit, we get $r_{x_{0}}^{*} \geq r_{0}$. Besides, (5.66) implies that $\lim \sup _{x \rightarrow r_{0}} \gamma_{x_{0}}^{*}(x) \leq \gamma_{r_{0}}^{z}\left(r_{0}\right)=z$, and the arbitrariness of $z \in\left(r_{0}, 2 r_{0}\right)$ leads to $\lim \sup _{x \rightarrow r_{0}} \gamma_{x_{0}}^{*}(x) \leq r_{0}$. Since $\gamma_{x_{0}}^{*}(x) \geq x$ for $x \in I_{x_{0}}^{*}$, we get $\lim _{x \rightarrow r_{0}} \gamma_{x_{0}}^{*}(x)=r_{0}$ and $r_{x_{0}}^{*} \leq r_{0}$. Hence, $r_{x_{0}}^{*}=r_{0} \leq \Gamma^{\infty}$, and, if $u\left(x_{0}\right)=\Gamma^{\infty}, r_{0} \geq u\left(x_{0}\right)$ implies that $r_{x_{0}}^{*}=\Gamma^{\infty}$.

(iii) Let $x_{1}$ in $\mathbf{D}^{-}$. Suppose that $x_{0}<x_{1}$ and that there exists $x_{2} \in I_{x_{0}}^{*} \cap I_{x_{1}}^{*}$. If ever $\gamma_{x_{0}}^{*}\left(x_{2}\right)=\gamma_{x_{1}}^{*}\left(x_{2}\right)$, the one-to-one property of the flow combined with the maximality of $I^{*}$ imply that $I_{x_{0}}^{*}=I_{x_{1}}^{*}$ and $\gamma_{x_{0}}^{*}=\gamma_{x_{1}}^{*}$ and conclude the proof. It therefore only remains to prove that $\gamma_{x_{0}}^{*}\left(x_{2}\right)=\gamma_{x_{1}}^{*}\left(x_{2}\right)$.

We assume on the contrary that $\gamma_{x_{0}}^{*}\left(x_{2}\right)<\gamma_{x_{1}}^{*}\left(x_{2}\right)$, the case where $\gamma_{x_{0}}^{*}\left(x_{2}\right)>\gamma_{x_{1}}^{*}\left(x_{2}\right)$ being treated similarly. The one-to-one property of the flow implies that $\gamma_{x_{0}}^{*}<\gamma_{x_{1}}^{*}$ on all the interval $I_{x_{0}}^{*} \cap I_{x_{1}}^{*}$. Furthermore, Lemma 5.3 (i) and Lemma 5.2 (i) ensure that $\lim _{r_{x_{1}}^{*}} \gamma_{x_{1}}^{*}=r_{x_{1}}^{*}$ and $\lim _{\ell_{x_{1}}^{*}} \gamma_{x_{1}}^{*}=\ell_{x_{1}}^{*}$. Hence, we deduce from the maximality of $I_{x_{1}}^{*}$ that $I_{x_{0}}^{*} \subset I_{x_{1}}^{*}$. Combining the definition of $z^{*}\left(x_{1}\right)$ with the continuity of the flow with respect to initial data, we obtain the existence of $z \in \mathbf{Z}\left(x_{1}\right)$ such that $z<z^{*}\left(x_{1}\right)$ and $\gamma_{x_{0}}^{*}\left(x_{2}\right)<$ $\gamma_{x_{1}}^{z}\left(x_{2}\right)<\gamma_{x_{1}}^{*}\left(x_{2}\right)$. Once again, the one-to-one property of the flow implies that $I_{x_{0}}^{*} \subset I_{x_{1}}^{z}$ and $\gamma_{x_{0}}^{*}<\gamma_{x_{1}}^{z}<\gamma_{x_{1}}^{*}$ on $I_{x_{0}}^{*}$. Since $z \in \mathbf{Z}\left(x_{1}\right)$, we deduce that $\gamma_{x_{1}}^{z}\left(x_{0}\right) \in \mathbf{Z}\left(x_{0}\right)$ while $\gamma_{x_{1}}^{z}\left(x_{0}\right)>z^{*}\left(x_{0}\right)=\gamma_{x_{0}}^{*}\left(x_{0}\right)$, which contradicts the definition of $z^{*}\left(x_{0}\right)$.

Finally, we are in position to provide the proof of Proposition 5.3:

Proof of Proposition 5.3 This construction follows similar ideas as in the proof of Proposition 5.1 in [5], but turns out to be simpler since $\Gamma^{\infty}<\infty$.

Let

$$
\mathcal{D}:=\bigcup_{x_{0} \in \mathbf{D}^{-}} I^{*}\left(x_{0}\right) \supset \mathbf{D}^{-} .
$$

Lemma 5.3 (iii) ensures that, for any $x_{0}$ and $x_{1}$ in $\mathbf{D}^{-}$, we either have $I_{x_{0}}^{*}=I_{x_{1}}^{*}$ or $I_{x_{0}}^{*} \cap I_{x_{1}}^{*}=\emptyset$. Hence, there exists a subset $\mathbf{D}_{0}^{-}$of $\mathbf{D}^{-}$such that $\mathcal{D}=\bigcup_{x_{0} \in \mathbf{D}_{0}^{-}} I^{*}\left(x_{0}\right)$ and, for any $x_{0}, x_{1} \in \mathbf{D}_{0}^{-}, x_{0} \neq x_{1}$ implies that $I_{x_{0}}^{*} \cap I_{x_{1}}^{*}=\emptyset$.

We now define the function $\gamma$ on $\mathbb{R}_{+} \backslash\{0\}$ by:

$$
\gamma(x):=\left\{\begin{array}{l}
\gamma_{x_{0}}^{*}(x) \text { if } x \in I_{x_{0}}^{*}, \text { for } x_{0} \in \mathbf{D}_{0}^{-} \\
x \text { otherwise }
\end{array}\right.
$$

According to Lemma 5.3, this definition does not depend on the choice of $\mathbf{D}_{0}^{-}$.

Lemmata 5.2 and 5.3 imply that $\gamma$ is continuous at the endpoints $\ell_{x_{0}}^{*}$ and $r_{x_{0}}^{*}$, for any $x_{0} \in \mathbf{D}_{0}^{-}$. Hence, setting $\gamma(0):=0$, we obtain a continuous function $\gamma$ on $\mathbb{R}_{+}$. For any 
$x_{0} \in \mathbf{D}_{0}^{-}, \gamma_{x_{0}}^{*}$ is increasing on $I_{x_{0}}^{*}$ and the identity function is increasing as well, so that $\gamma$ is increasing on $\mathbb{R}_{+}$. We now justify each assertion of the proposition separately. (i) is immediate from the definition of $\gamma$.

To prove (ii), we first notice that $\{x \geq 0: \gamma(x)=x\}=\mathbb{R}_{+} \backslash \mathcal{D} \subset \mathbb{R}_{+} \backslash \mathbf{D}^{-}$, so that $\mathcal{L} g(x, x) \geq 0$ on the set $\{x>0: \gamma(x)=x\}$. On the set $\{x>0: \gamma(x)>x\}$, since $\gamma$ has a positive derivative by Lemma 5.3 (ii) and satisfies (5.59), we have $\mathcal{L} g(x, \gamma(x))>0$. Finally, (iii) can be deduced from Lemma 5.3 (ii), since $r_{x_{0}}^{*} \leq \Gamma^{\infty}$ for any $x_{0} \in \mathbf{D}_{0}^{-}$.

\section{References}

[1] M. Dai, H. Jin, Y. Zhong and X. Zhou (2010). Buy low and sell high. Contemporary Quantitative Finance: Essays in Honour of Eckhard Platen, C Chiarella \& A. Novikov Editors, , 317-334.

[2] J. Du Toit and G. PEskir (2007). The trap of complacency in predicting the maximum. Ann. Probab. 35, 340-365.

[3] J. Du Toit and G. Peskir (2008). Predicting the time of the ultimate maximum for Brownian motion with drift. Proc. Math. Control Theory Finance (Lisbon 2007), 95-112, Springer Berlin.

[4] J. Du Toit and G. Peskir (2009). Selling a stock at the ultimate maximum. Ann. Appl. Probab. 19 (3), 983-1014.

[5] G.E. Espinosa and N. Touzi (2010), Detecting the maximum of a scalar diffusion with negative drift, preprint.

[6] S.E. Graversen, G. Peskir and A.N. Shiryaev (2001). Stopping Brownian motion without anticipation as close as possible to its ultimate maximum. Theory Probab. Appl. 45 (125-136).

[7] D. Hobson (2007). Optimal stopping of the maximum process: a converse to the results of Peskir. Stochastics 79 (1), 85-102.

[8] G. Peskir (1998). Optimal stopping of the maximum process: the maximality principle. Ann. Probab. Vol. 26, No. 4 (1614-1640).

[9] A.N. Shiryaev, Z. Xu and X. Zhou (2008). Thou shalt buy and hold. Quantitative Finance 8, 765-776.

[10] M.A. Urusov (2005). On a property of the moment at which Brownian motion attains its maximum and some optimal stopping problems. Theory Probab. Appl. 49 (169-176). 